\title{
Highly Active in situ Catalysts for anti-Markovnikov Hydration of Terminal Alkynes
}

Aurélie Labonne, Thomas Kribber and Lukas Hintermann*

Institute of Organic Chemistry, RWTH Aachen University, Landoltweg 1, D-52074 Aachen, Germany lukas.hintermann@oc.rwth-aachen.de

\section{Supporting Information}

Contents 1. General Information

2. General Procedures for Hydration Experiments

3. Synthesis and Characterization of 2-Aryl-6-bromo-pyridines (P)

4. Synthesis and Characterization of 2-Aryl-6-diphenylphosphinopyridines (L)

5. New Substances from Alkyne Hydration

6. Selected NMR Spectra

\section{General Information}

Syntheses of and with air-sensitive materials were performed under argon, using freshly distilled and degassed solvents.

Analytical data were measured by in-house services at the Institute of Organic Chemistry, RWTH Aachen University. Melting points (M.p.) were measured with a digital thermometer, therefore "corrected" values. MS: $m / z$ (\% relative abundance). ${ }^{1} \mathbf{H}-\mathbf{N M R}: \delta / \mathrm{ppm}, J / \mathrm{Hz}$, referenced to tetramethylsilane. ${ }^{13} \mathbf{C}-\mathbf{N M R}: \delta / \mathrm{ppm}, J / \mathrm{Hz}$, referenced to tetramethylsilane. ${ }^{31} \mathbf{P}-\mathbf{N M R}: \delta / \mathrm{ppm}, J / \mathrm{Hz}$. IR: $v / \mathrm{cm}^{-1}$, intensity: $\mathrm{s}=$ strong, $\mathrm{m}=$ medium, $\mathrm{w}=$ weak.

The following substances were prepared according to literature procedures: 2-Chloro-4,6-diphenyl-1,3-

diazine $(4)^{1}$, 2-chloro-6-phenyl-pyridine and 6-phenyl-2-diphenylphosphinopyridine (L1). ${ }^{2} \mathrm{NaPPh}_{2}$ was prepared by the $\mathrm{Na} / \mathrm{NH}_{3} / \mathrm{PPh}_{3}$ method ${ }^{3}$ and careful dissolution of the residue of evaporation in THF (0.7-1 M). A solution of $\mathrm{LiPPh}_{2}$ was generated from $\mathrm{HPPh}_{2}($ Strem) and BuLi in THF. 


\section{General Procedures for Hydration Experiments}

2.1. General procedure for anti-Markovnikov hydration of terminal alkynes (preparative): A Schlenk flask was charged with $\left[\mathrm{CpRu}\left(\eta^{6}\right.\right.$-naphthalene $\left.)\right] \mathrm{PF}_{6}(\mathbf{C} ; 1-5 \mathrm{~mol} \%)$, pyridyl-phosphane ligand $\mathbf{L}$ (2-10 mol \%, 2 equiv relative to $\mathbf{C}$ ) and $\mathrm{CH}_{3} \mathrm{CN}$ (analytical reagent, degassed, $1 \mathrm{~mL} / 10 \mathrm{mg} \mathbf{C}$ ). The mixture was heated to $60^{\circ} \mathrm{C}$ for $1-6 \mathrm{~h}$ ( $1 \mathrm{~h}$ for $\mathbf{L 1}$; $3 \mathrm{~h}$ for $\mathbf{L 2}, \mathbf{L 3} ; 6 \mathrm{~h}$ for $\left.\mathbf{L 5}, \mathbf{L 6}, \mathbf{L} 7\right)$, then the solvent was removed in vacuo to afford a yellow powder or resin. (This in situ-catalyst is storable). A solution of the alkyne (100 mol \%) and water (5 equiv relative to alkyne) in acetone (1-4 mL/mmol substrate) was added to the catalyst and the resulting solution heated to $55-60{ }^{\circ} \mathrm{C}$. After completion of the reaction (1.5 - $15 \mathrm{~h}$, according to GC-MS or TLC), the solution was allowed to cool to room temperature and the solvent removed in vacuo to afforded the crude aldehydes, which were purified by either Kugelrohr distillation or flash column chromatography $\left(\mathrm{SiO}_{2}, t\right.$-BuOMe/hexanes).

\subsection{General procedure for anti-Markovnikov hydration with several ligands (kinetic runs): A}

Schlenk flask was charged with $\left[\mathrm{CpRu}\left(\eta^{6}\right.\right.$-naphthalene $\left.)\right] \mathrm{PF}_{6}(\mathbf{C} ; 5 \mathrm{~mol} \%)$ and the aza-aryl-phosphane ligand $\mathbf{L}$ ( 2 equiv relative to $\mathbf{C}, 10 \mathrm{~mol} \%$ ) and the in situ catalyst prepared as above. After removal of the solvent, the residual yellow powder or resin was carefully dried for $1 \mathrm{~h}$ in a high vacuum. Subsequently, a solution of 1-octyne ( $1 \mathrm{mmol}, 1$ equiv), $\mathrm{H}_{2} \mathrm{O}(5 \mathrm{mmol}, 5$ equiv) and tetradecane (80 $\mathrm{mg}$ ) in acetone $(4 \mathrm{~mL})$ was added and the resulting yellow solution kept at $45^{\circ} \mathrm{C}$ ( or $60{ }^{\circ} \mathrm{C}$ ). Aliquots were removed at regular intervals to monitor the progress of the reaction by GC-analysis (FIDdetection), based on separately determined response factors of the tetradecane standard relative to octyne and octanal. 


\section{Synthesis and Characterization of 2-Aryl-6-bromo-pyridines (P)}

\subsection{General procedure for Ni-catalyzed cross-coupling of aryl Grignard reagents and 2,6-} dibromopyridine (3). A solution of the Grignard reagent (1.1-1.5 equiv) in THF was slowly added to a solution of 2,6-dibromopyridine (1.0 equiv) and $\left[\mathrm{NiCl}_{2}(\mathrm{PCy})_{3}\right](0.2-1 \mathrm{~mol} \%)$ in dry $\mathrm{THF}$ at room temperature. The reaction mixture was stirred at room temperature for 7-30 h, then quenched by careful addition $\mathrm{NH}_{4} \mathrm{Cl}$ (aq). The aqueous phase was extracted with $t$ - $\mathrm{BuOMe}$ or $\mathrm{CH}_{2} \mathrm{Cl}_{2}$. The combined organic phases were washed with water and dried over $\mathrm{MgSO}_{4}$. Evaporation afforded crude products, which were purified by either crystallization or flash column chromatography $\left(\mathrm{SiO}_{2}\right)$.

\subsection{Substance data}

2-Bromo-6-mesityl-pyridine (P3). Prepared according to the general procedure.

This material contained some 2,6-dimesityl-pyridine as impurity and was used in the phosphination step without further purification.

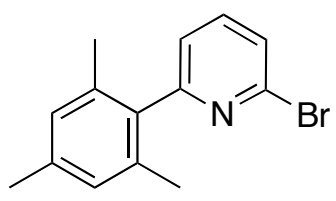

H-NMR (300 MHz, $\left.\mathrm{CDCl}_{3}\right): 2.03(\mathrm{~s}, 6 \mathrm{H}), 2.30(\mathrm{~s}, 3 \mathrm{H}), 6.91($ br s, $2 \mathrm{H}), 7.17(\mathrm{~d}, \mathrm{~J}=7.6 \mathrm{~Hz}, 1 \mathrm{H})$, $7.43(\mathrm{~d}, \mathrm{~J}=7.5,1 \mathrm{H}), 7.5(\mathrm{t}, \mathrm{J} \approx 7.5,1 \mathrm{H}) .{ }^{13} \mathbf{C}-\mathbf{N M R}\left(75 \mathrm{MHz}, \mathrm{CDCl}_{3}\right): 20.2\left(\mathrm{CH}_{3}\right), 21.1\left(\mathrm{CH}_{3}\right), 123.7$ $(\mathrm{CH}), 126.0(\mathrm{CH}), 128.4(\mathrm{CH}), 135.6(\mathrm{C}), 136.3(\mathrm{C}), 137.9(\mathrm{C}), 138.5(\mathrm{CH}), 141.8(\mathrm{C}), 161.2(\mathrm{C})$. IR (KBr): 3008m, 2960m, 1730m, 1610s, 1572s, 1440s, 1382s, 1277m, 1159s, 1121s, 1034s, 849s, 794s, 745s. MS (EI +): 275/277 (51, M+1), 274/276 (100, [M-H] $\left.]^{+}\right), 196$ (32), 181 (18), 90 (19). HRMS (EI): Calcd for $\mathrm{C}_{14} \mathrm{H}_{14} \mathrm{BrN}$ (276.17): 275.030972, found 275.030934.

2-Bromo-6-(2,6-di-isopropoxy-phenyl)-pyridine (P4). According to the general procedure, with the following modifications: The Grignard reagent was prepared by ortho-metallation of 1,3-diisopropoxy-benzene with $\mathrm{n}$-BuLi in hexane at $50^{\circ} \mathrm{C}$ for

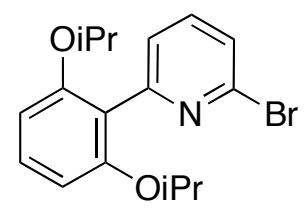
$10 \mathrm{~h}$, followed by addition of a THF-solution of $\mathrm{MgBr}_{2}$. Mp.: 77-78 ${ }^{\circ} \mathrm{C} . \mathbf{H}-\mathbf{N M R}\left(300 \mathrm{MHz}, \mathrm{CDCl}_{3}\right)$ : $1.18(\mathrm{~d}, \mathrm{~J}=6.1,12 \mathrm{H}), 4.42(\mathrm{sept}, \mathrm{J}=6.1,2 \mathrm{H}), 6.59(\mathrm{~d}, \mathrm{~J}=8.4,2 \mathrm{H}), 7.22(\mathrm{~d}, \mathrm{~J}=8.4,1 \mathrm{H}), 7.27(\mathrm{~d}, \mathrm{~J}=$ 7.5, $1 \mathrm{H}), 7.37$ (d, J = 7.8, $1 \mathrm{H}), 7.53$ (t, J = 7.7, $1 \mathrm{H}) .{ }^{13} \mathbf{C}-\mathbf{N M R}\left(75 \mathrm{MHz}, \mathrm{CDCl}_{3}\right): 22.1(\mathrm{CH} 3), 71.4$ $(\mathrm{CH}), 107.5(\mathrm{CH}), 120.9(\mathrm{C}), 125.3(\mathrm{CH}), 125.4(\mathrm{CH}), 129.7(\mathrm{CH}), 137.5(\mathrm{CH}), 140.5(\mathrm{C}), 156.2(\mathrm{C})$, 156.9 (C). IR (KBr): 2976m, 2921m, 1592s, 1545m, 1459s, 1381m, 1249s, 1118s, 1062s, 785m. MS (EI+): 349/351 (33, M+), 334/336 (11), 265/267 (100), 249/251 (25), 186 (14). EA calcd for $\mathrm{C}_{17} \mathrm{H}_{20} \mathrm{BrNO}_{2}(350.25)$ C 58.30, H 5.76, N 4.00, found C 58.43, H 5.82, N 4.11. 
2-Bromo-6-(2,6-diisopropylphenyl)-pyridine (P5). According to the general procedure. White solid. Mp: $176-177^{\circ} \mathrm{C} .{ }^{1} \mathbf{H}-\mathbf{N M R}\left(400 \mathrm{MHz}, \mathrm{CDCl}_{3}\right): 1.09$ (d, $J=$ $\left.6.9,6 \mathrm{H}, \mathrm{CH}_{3}\right), 1.14\left(\mathrm{~d}, J=6.9,6 \mathrm{H}, \mathrm{CH}_{3}\right), 2.47$ (sept, $\left.J=6.9,2 \mathrm{H}, \mathrm{CH}\right), 7.21(\mathrm{~d}, \mathrm{~J}$

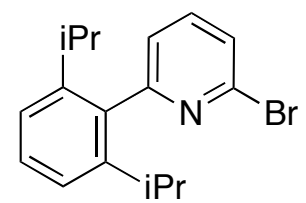
$=7.8,2 \mathrm{H}, \mathrm{Ar}-\mathrm{H}), 7.23(\mathrm{dd}, J=7.4,0.8,1 \mathrm{H}, \mathrm{Ar}-\mathrm{H}), 7.36(\mathrm{t}, J=7.7,1 \mathrm{H}, \mathrm{Ar}-\mathrm{H}), 7.48(\mathrm{dd}, J=8.0,0.8$, $1 \mathrm{H}, \mathrm{Ar}-\mathrm{H}), 7.60$ (t, $J=7.4,1 \mathrm{H}, \mathrm{Ar}-\mathrm{H}) .{ }^{13} \mathbf{C}-\mathbf{N M R}\left(100 \mathrm{MHz}, \mathrm{CDCl}_{3}\right):$ 22.7, 23.0, 29.3, 121.5, 122.6, 124.8, 127.7, 135.9, 136.7, 140.3, 145.1, 159.7. IR (KBr): 3028 (s), 2956 (m), 1569 (s), 1431 (s), 1113

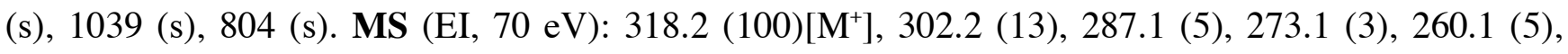
238.2 (23), 196.2 (21), 180.2 (10). HRMS (EI) calcd for $\mathrm{C}_{17} \mathrm{H}_{20} \mathrm{BrN}$ : 317.077923, found: 317.077795.

2-Bromo-6-(2,4,6-triisopropylphenyl)-pyridine (P6). ${ }^{4}$ Prepared according to the general procedure as white solid. Due to low solubility of the product, the reaction needed efficient stirring. Recrystallization from toluene. Mp: $241{ }^{\circ} \mathrm{C}$.

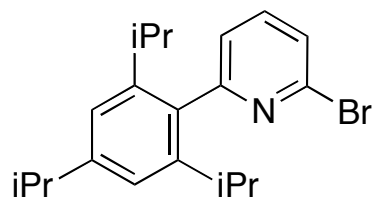
${ }^{1}$ H-NMR (300 MHz, $\left.\mathrm{CDCl}_{3}\right): 1.09\left(\mathrm{~d}, J=6.9,6 \mathrm{H}, \mathrm{CH}_{3}\right), 1.14\left(\mathrm{~d}, J=6.9,6 \mathrm{H}, \mathrm{CH}_{3}\right), 1.26(\mathrm{~d}, J=6.9,6$ $\mathrm{H}, \mathrm{CH}_{3}$ ), 2.47 (sept, $\left.J=6.9,2 \mathrm{H}, \mathrm{CH}\right), 2.91$ (sept, $\left.J=6.9,1 \mathrm{H}, \mathrm{CH}\right), 7.05$ (s, $\left.2 \mathrm{H}, \mathrm{Ar}-\mathrm{H}\right), 7.25$ (dd, $J=$ 7.8, 0.9, $1 \mathrm{H}, \mathrm{Ar}-\mathrm{H}), 7.46$ (dd, $J=7.9,1.0,1 \mathrm{H}, \mathrm{Ar}-\mathrm{H}), 7.59$ (t, $J=7.4,1 \mathrm{H}, \mathrm{Ar}-\mathrm{H}) .{ }^{13} \mathbf{C}-\mathbf{N M R}(75$ $\left.\mathrm{MHz}, \mathrm{CDCl}_{3}\right)$ : 23.8, 24.0, 24.1, 30.4, 34.4, 120.8, 123.9, 125.9, 134.9, 137.9, 141.5, 146.1, 149.3, 161.3. IR (KBr): 2961 (m), 2868 (m), 1574 (s), 1544 (s), 1432 (s), 1127 (s). MS (EI, 70 eV): 361.1 (53) $\left[\mathrm{M}^{+}\right], 360.1$ (100), 359.1 (59), 344,1 (8). HRMS (EI): calcd for $\mathrm{C}_{20} \mathrm{H}_{26} \mathrm{BrN}$ : 359.12487, Found: 359.12486.

2-Bromo-6-(2,4,6-triphenyl-phenyl)-pyridine (P7). The compound was prepared according to the general procedure with the following modifications: $1 \mathrm{~mol} \%$ of Ni-complex as catalyst, the reaction was stirred for $4 \mathrm{~d}$ at $75^{\circ} \mathrm{C}$,

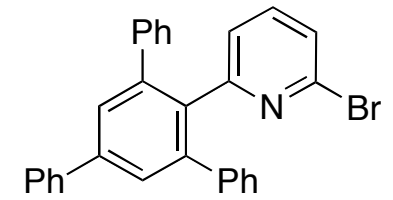
then for $4 \mathrm{~d}$ at $85^{\circ} \mathrm{C}$. CC (hexanes/toluene 3:2-1:1 - toluene). Yield 57\% of a white crystalline powder. Mp.: $243-244^{\circ} \mathrm{C} . \mathbf{H}-\mathbf{N M R}\left(400 \mathrm{MHz}, \mathrm{CDCl}_{3}\right): 6.82(\mathrm{~d}, \mathrm{~J}=7.3,1 \mathrm{H}), 7.12(\mathrm{t}, \mathrm{J} \approx 7.0,1 \mathrm{H}), 7.15-7.28$ $(\mathrm{m}, 11 \mathrm{H}), 7.38(\mathrm{t}, \mathrm{J}=7.3,1 \mathrm{H}), 7.46(\mathrm{t}, \mathrm{J} \approx 7.5,2 \mathrm{H}), 7.65-7.72(\mathrm{~m}, 4 \mathrm{H}) .{ }^{13} \mathbf{C}-\mathbf{N M R}(100 \mathrm{MHz}$, $\left.\mathrm{CDCl}_{3}\right): 125.1(\mathrm{CH}), 125.3(\mathrm{CH}), 126.5(\mathrm{CH}), 127.1(\mathrm{CH}), 127.5(\mathrm{CH}), 127.6(\mathrm{CH}), 128.0(\mathrm{CH}), 128.7$ (CH), 129.5 (CH), 135.7 (C), 137.0 (CH), 140.1 (C), 140.3 (C), 140.9 (C), 142.3 (C), 159.5 (C). IR (KBr): 3031w, 1699m, 1651m, 1575m, 1558s, 1542s, 1506m, 1420m, 1129m, 767s, 698s. MS (EI+): 461/463 (60, M+), 460/462 (50), 383 (28), 382 (100), 354 (14), 341 (11), 183 (7). EA calcd for $\mathrm{C}_{29} \mathrm{H}_{20} \mathrm{BrN}$ (462.38): C 75.33, H 4.36, N 3.03, found C 75.64 H 4.60, N 3.01. 


\section{Synthesis and Characterization of 2-Aryl-6-diphenylphosphinopyridines (L)}

4.1. General procedure. A solution of $\mathrm{NaPPh}_{2}$ (1.0 equiv) in $\mathrm{THF}$ was added dropwise to a solution of 2-aryl-6-bromopyridine (P, 1.0 equiv) in $\mathrm{Et}_{2} \mathrm{O} /$ toluene $(3: 2)$ at $0^{\circ} \mathrm{C}$. The resulting orange-yellow mixture was stirred at $0{ }^{\circ} \mathrm{C}$ for $1.5 \mathrm{~h}-1.5 \mathrm{~d}$. The reaction was diluted with $\mathrm{CH}_{2} \mathrm{Cl}_{2}(1 / 3$ of the reaction volume) and the resulting suspension filtered through a plug of Celite. Removal of the solvent in vacuum afforded a crude product that was purified by flash column chromatography under argon $\left(\mathrm{CH}_{2} \mathrm{Cl}_{2} /\right.$ hexanes, $\left.\mathrm{SiO}_{2}\right)$ or recrystallization from $\mathrm{MeOH}$.

6-(2,4,6-trimethylphenyl-2-diphenylphosphino-pyridine (L3). White solid. Mp: $118{ }^{\circ} \mathrm{C} .{ }^{1} \mathbf{H}-\mathbf{N M R}\left(300 \mathrm{MHz}, \mathrm{CDCl}_{3}\right): 1.96\left(\mathrm{~s}, 6 \mathrm{H}, \mathrm{CH}_{3}\right), 2.28(\mathrm{~s}, 3 \mathrm{H}$, $\mathrm{CH}_{3}$ ), 6.87 (s, $\left.2 \mathrm{H}, \mathrm{Ar}-\mathrm{H}\right), 6.97$ (dt, $\left.J=7.7,0.5,1 \mathrm{H}, \mathrm{Ar}-\mathrm{H}\right), 7.09$ (dt, $J=7.7$,

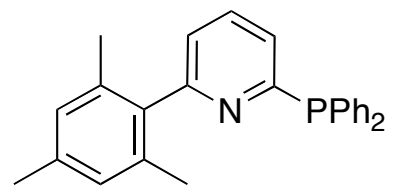
1.0, $1 \mathrm{H}, \mathrm{Ar}-\mathrm{H}), 7.31-7.43(\mathrm{~m}, 10 \mathrm{H}, \mathrm{Ar}-\mathrm{H}), 7.59$ (td, $J=6.9,2.0,1 \mathrm{H}, \mathrm{Ar}-\mathrm{H}) .{ }^{13} \mathrm{C}-\mathbf{N M R}(75 \mathrm{MHz}$, $\left.\mathrm{CDCl}_{3}\right): 20.3\left(\mathrm{CH}_{3}\right), 21.1\left(\mathrm{CH}_{3}\right), 123.3(\mathrm{CH}), 125.4\left(\mathrm{~d}, J_{\mathrm{PC}}=14, \mathrm{CH}\right), 128.2(\mathrm{CH}), 128.5\left(\mathrm{~d}, J_{\mathrm{PC}}=7\right.$, $\mathrm{CH}), 128.9(\mathrm{CH}), 134.3\left(\mathrm{~d}, J_{\mathrm{PC}}=20.2, \mathrm{CH}\right), 135.7(\mathrm{C}), 135.8\left(\mathrm{~d}, J_{\mathrm{PC}}=2, \mathrm{CH}\right), 136.5\left(\mathrm{~d}, J_{\mathrm{PC}}=10.5, \mathrm{C}\right)$, $137.4(\mathrm{C}), 137.7(\mathrm{C}), 160.6$ (d, $\left.J_{\mathrm{PC}}=13, \mathrm{C}\right), 164.0\left(\mathrm{~d}, J_{\mathrm{PC}}=5, \mathrm{C}\right) .{ }^{31} \mathbf{P}-\mathbf{N M R}\left(121 \mathrm{MHz}, \mathrm{CDCl}_{3}\right):-4.7$ (s). IR (KBr): 3466 (m), 2917 (m), 1569 (s), 1549 (s), 1440 (s), 853 (s), 757 (s), 695 (s). MS (EI, 70 eV): 381.1 (100)[ $\left.\mathrm{M}^{+}\right], 302.1$ (28), 287.0 (5), 195.1 (9), 183.0 (6). EA calcd for $\mathrm{C}_{26} \mathrm{H}_{24} \mathrm{NP}: \mathrm{C}$ 81.87, H 6.34, N 3.67, found: C 82.06, H 6.03, N 3.66.

6-(2,6-Diisopropoxy-phenyl)-2-diphenylphosphino-pyridine (L4). White solid. Mp: $112{ }^{\circ} \mathrm{C} .{ }^{1} \mathbf{H}-\mathbf{N M R}\left(400 \mathrm{MHz}, \mathrm{C}_{6} \mathrm{D}_{6}\right): \delta=0.96\left(\mathrm{~d}, J=6.0,12 \mathrm{H}, \mathrm{CH}_{3}\right), 4.16$ (sept, $J=6.0,2 \mathrm{H}, \mathrm{CH}), 6.51(\mathrm{~d}, J=8.5,2 \mathrm{H}, \mathrm{Ar}-\mathrm{H}), 6.99(\mathrm{dt}, J=7.5,1.2,1 \mathrm{H}$,

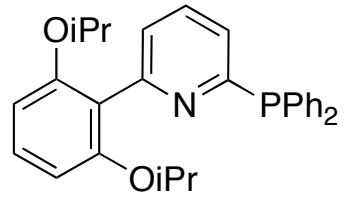
Ar-H), 7.02-7.16 (m, 9 H, Ar-H), 7.52 (td, $J=7.6,1.6,4$ H, Ar-H). ${ }^{13}$ C-NMR $\left(100 \mathrm{MHz}, \mathrm{C}_{6} \mathrm{D}_{6}\right): 22.1$ $\left(\mathrm{CH}_{3}\right), 71.3(\mathrm{CH}), 108.1(\mathrm{CH}), 123.9(\mathrm{C}), 125.8\left(\mathrm{~d}, J_{\mathrm{PC}}=17, \mathrm{CH}\right), 128.4\left(\mathrm{~d}, J_{\mathrm{PC}}=7, \mathrm{CH}\right), 128.5(\mathrm{CH})$, $129.0(\mathrm{CH}), 134.5\left(\mathrm{~d}, J_{\mathrm{PC}}=20, \mathrm{CH}\right), 134.7\left(\mathrm{~d}, J_{\mathrm{PC}}=3, \mathrm{CH}\right), 138.0\left(\mathrm{~d}, J_{\mathrm{PC}}=13.0, \mathrm{C}\right), 156.4\left(\mathrm{~d}, J_{\mathrm{PC}}=13\right.$, C), $157.3(\mathrm{C}), 162.6\left(\mathrm{~d}, J_{\mathrm{PC}}=5, \mathrm{C}\right) .{ }^{31} \mathbf{P}-\mathbf{N M R}\left(160 \mathrm{MHz}, \mathrm{C}_{6} \mathrm{D}_{6}\right):-4.8(\mathrm{~s}) . \mathbf{I R}(\mathrm{KBr}): v=2971(\mathrm{~m})$, 1583 (s), 1451 (s), 1373 (s), 1238 (s), 1114 (s), 1047 (s), 742 (s), 695 (m). MS (EI+): 455.2 (100)[M+], 440.2 (49), 412.2 (16), 396.2 (13), 382.2 (19), 370.1 (41), 354.1 (28), 338.1 (30), 292.0 (22), 254.0 (32). EA calcd for $\mathrm{C}_{29} \mathrm{H}_{30} \mathrm{NO}_{2} \mathrm{P}: \mathrm{C}$ 76.46, $\mathrm{H}$ 6.64, N 3.07, found: C 76.03, H 6.70, N 3.05. 
6-(2,6-Diisopropyl-phenyl)-2-diphenylphosphino-pyridine (L5). White solid. Mp: $143-144{ }^{\circ} \mathrm{C} .{ }^{1} \mathbf{H}-\mathbf{N M R}\left(400 \mathrm{MHz}, \mathrm{C}_{6} \mathrm{D}_{6}\right): 1.03\left(\mathrm{~d}, J=6.8,6 \mathrm{H}, \mathrm{CH}_{3}\right), 1.09$ $\left(\mathrm{d}, J=6.8,6 \mathrm{H}, \mathrm{CH}_{3}\right), 2.66(\mathrm{sept}, J=6.9,2 \mathrm{H}, \mathrm{CH}), 6.86(\mathrm{dt}, J=7.4,0.9,1 \mathrm{H}$,

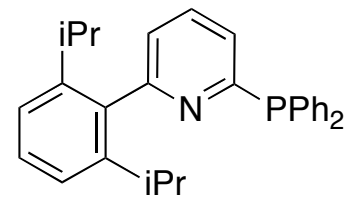
Ar-H), 7.00 (td, $J=7.7,2.2,1$ H, Ar-H), 7.03-7.10 (m, 7 H, Ar-H), 7.15 (d, J = 7.8, 2 H, Ar-H), 7.29 (t, $J=7.7,1 \mathrm{H}, \mathrm{Ar}-\mathrm{H}), 7.51(\mathrm{td}, J=7.7,1.6,4 \mathrm{H}, \mathrm{Ar}-\mathrm{H}) .{ }^{13} \mathbf{C}-\mathbf{N M R}\left(100 \mathrm{MHz}, \mathrm{C}_{6} \mathrm{D}_{6}\right): 24.0\left(\mathrm{CH}_{3}\right), 24.4$ $\left(\mathrm{CH}_{3}\right), 30.7(\mathrm{CH}), 122.7(\mathrm{CH}), 123.3(\mathrm{CH}), 125.9\left(\mathrm{~d}, J_{\mathrm{PC}}=20, \mathrm{CH}\right), 128.5\left(\mathrm{~d}, J_{\mathrm{PC}}=7, \mathrm{CH}\right), 128.7(\mathrm{CH})$, $128.8(\mathrm{CH}), 134.4\left(\mathrm{~d}, J_{\mathrm{PC}}=19.9, \mathrm{CH}\right), 135.1\left(\mathrm{~d}, J_{\mathrm{PC}}=3.8, \mathrm{CH}\right), 137.2\left(\mathrm{~d}, J_{\mathrm{PC}}=11.4, \mathrm{C}\right), 139.0(\mathrm{C})$, $146.4(\mathrm{C}), 160.8\left(\mathrm{~d}, J_{\mathrm{PC}}=11.5, \mathrm{C}\right), 163.9\left(\mathrm{~d}, J_{\mathrm{PC}}=1.4, \mathrm{C}\right) .{ }^{31} \mathbf{P}-\mathbf{N M R}\left(160 \mathrm{MHz}, \mathrm{C}_{6} \mathrm{D}_{6}\right):-4.3$ (s). IR (KBr): 3055 (s), 2957 (s), 2865 (s), 1554 (s), 1433 (s), 744 (s), 693 (s). MS (EI+): 423.3 (100)[M+], 408.2 (5), 344.1 (11), 328.1 (6), 236.1 (20), 222.1 (38), 211.5 (6). HRMS calcd for $\mathrm{C}_{29} \mathrm{H}_{30} \mathrm{BrN}$ 423.211589, found: 423.211409 .

6-(2,4,6-Triisopropyl-phenyl)-2-diphenylphosphino-pyridine (L6). White solid. Mp: $198{ }^{\circ} \mathrm{C} .{ }^{1} \mathbf{H}-\mathbf{N M R}\left(400 \mathrm{MHz}, \mathrm{C}_{6} \mathrm{D}_{6}\right): 1.10\left(\mathrm{~d}, J=6.9,6 \mathrm{H}, \mathrm{CH}_{3}\right)$, $1.17\left(\mathrm{~d}, J=6.9,6 \mathrm{H}, \mathrm{CH}_{3}\right), 1.28\left(\mathrm{~d}, J=6.9,6 \mathrm{H}, \mathrm{CH}_{3}\right), 2.73$ (sept, $J=6.9,2$

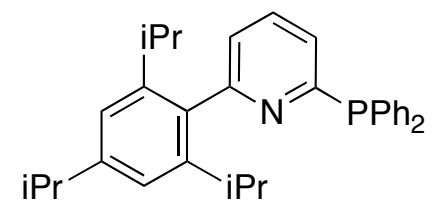
H, CH), 2.86 (sept, $J=6.9,1 \mathrm{H}, \mathrm{CH}), 6.91$ (dt, $J=7.4,1.2,2 \mathrm{H}, \mathrm{Ar}-\mathrm{H}), 6.99$ (td, $J=7.7,2.2,1 \mathrm{H}$, ArH), 7.03-7.10 (m, $6 \mathrm{H}, \mathrm{Ar}-\mathrm{H}), 7.19$ (s, $2 \mathrm{H}, \mathrm{Ar}-\mathrm{H}), 7.50-7.57$ (m, $4 \mathrm{H}, \mathrm{Ar}-\mathrm{H}) .{ }^{13} \mathbf{C}-\mathbf{N M R}(100 \mathrm{MHz}$, $\left.\mathrm{C}_{6} \mathrm{D}_{6}\right): 23.9\left(\mathrm{CH}_{3}\right), 24.2\left(\mathrm{CH}_{3}\right), 24.2\left(\mathrm{CH}_{3}\right), 30.7(\mathrm{CH}), 34.7(\mathrm{CH}), 120.5(\mathrm{CH}), 123.3(\mathrm{CH}), 125.7(\mathrm{~d}$, $\left.J_{\mathrm{PC}}=20.1, \mathrm{CH}\right), 128.3\left(\mathrm{~d}, J_{\mathrm{PC}}=7, \mathrm{CH}\right), 128.6(\mathrm{CH}), 134.3\left(\mathrm{~d}, J_{\mathrm{PC}}=20, \mathrm{CH}\right), 134.9\left(\mathrm{~d}, J_{\mathrm{PC}}=3.5, \mathrm{CH}\right)$, $136.8(\mathrm{C}) 137.1\left(\mathrm{~d}, J_{\mathrm{PC}}=13, \mathrm{C}\right), 146.3(\mathrm{C}), 148.6(\mathrm{C}), 160.9\left(\mathrm{~d}, J_{\mathrm{PC}}=12, \mathrm{C}\right), 163.7\left(\mathrm{~d}, J_{\mathrm{PC}}=2, \mathrm{C}\right) .{ }^{31} \mathbf{P}-$ NMR (160 MHz, C $6 \mathrm{D}_{6}$ ): -4.3 (s). IR (KBr): 3437 (m), 2959 (s), 2868 (s), 1565 (s), 1435 (s), 745 (s).

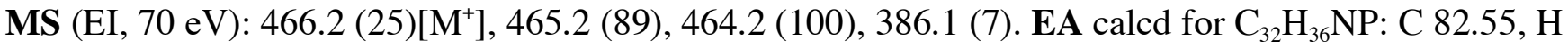
7.79, N 3.01, found: C 82.20, H 7.79, N 2.91.

6-(2,4,6-Triphenyl-phenyl)-2-diphenylphosphino-pyridine (L7). White solid. Mp: $197-198{ }^{\circ} \mathrm{C} .{ }^{1} \mathbf{H}-\mathbf{N M R}\left(400 \mathrm{MHz}, \mathrm{C}_{6} \mathrm{D}_{6}\right)$ : 6.57-6.65 (m, $\left.2 \mathrm{H}, \mathrm{Ar}-\mathrm{H}\right)$, 6.82 (ddd, $J=6.6,2.7,1.9,1 \mathrm{H}, \mathrm{Ar}-\mathrm{H}), 6.96-7.10$ (m, $13 \mathrm{H}, \mathrm{Ar}-\mathrm{H}), 7.19$ -

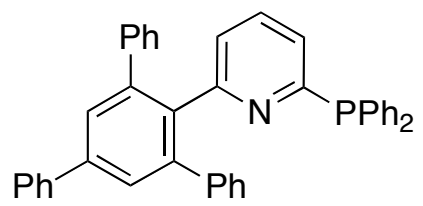
7.24 (m, $6 \mathrm{H}, \mathrm{Ar}-\mathrm{H}), 7.38$ (td, $J=7.7,2.0,4 \mathrm{H}, \mathrm{Ar}-\mathrm{H}), 7.45$ (dt, J=6.8, 1.6, 2 H, Ar-H), 7.63 (s, $2 \mathrm{H}$, Ar-H). ${ }^{13}$ C-NMR (75 MHz, $\left.\mathrm{C}_{6} \mathrm{D}_{6}\right): 125.5(\mathrm{CH}), 126 .\left(\mathrm{d}, J_{\mathrm{PC}}=26, \mathrm{CH}\right), 126.7(\mathrm{CH}), 127.7(\mathrm{CH}), 127.7$ $(\mathrm{CH}), 128.0(\mathrm{CH}), 128.5(\mathrm{CH}), 128.7\left(\mathrm{~d}, J_{\mathrm{PC}}=7, \mathrm{CH}\right), 128.8(\mathrm{CH}), 129.1(\mathrm{CH}), 130.3(\mathrm{CH}), 134.6(\mathrm{~d}$, $\left.J_{\mathrm{PC}}=5, \mathrm{CH}\right), 134.7\left(\mathrm{~d}, J_{\mathrm{PC}}=20, \mathrm{CH}\right), 137.8\left(\mathrm{~d}, J_{\mathrm{PC}}=12, \mathrm{C}\right), 138.5(\mathrm{C}), 140.9(\mathrm{C}), 141.4(\mathrm{C}), 142.2(\mathrm{C})$, $142.9(\mathrm{C}), 160.1\left(\mathrm{~d}, J_{\mathrm{PC}}=10, \mathrm{C}\right), 162.5$ (C). ${ }^{31} \mathbf{P}-\mathbf{N M R}\left(160 \mathrm{MHz}, \mathrm{C}_{6} \mathrm{D}_{6}\right):-3.1$ (s). IR (KBr): 3437 (m), 
S 7

3050 (s), 1688 (s), 1557 (s), 1420 (m), 1150 (s), 693 (m). MS (EI, $70 \mathrm{eV}): 567.3$ (100)[M+1, 490.2 (25), 412.1 (4), 283.6 (45). EA calcd for $\mathrm{C}_{41} \mathrm{H}_{30} \mathrm{NP}$ : C 86.75, H 5.33, N 2.47, found C 87.10, H 5.36, N 2.28. HRMS: Calcd for $\mathrm{C}_{41} \mathrm{H}_{30} \mathrm{NP}: 567.211589$, found: 567.211506.

4,6-Diphenyl-2-diphenylphosphino-1,3-diazine (L8). Prepared according to the general procedure, white solid. Mp: $104-105{ }^{\circ} \mathrm{C}$. ${ }^{1} \mathbf{H}-\mathbf{N M R}\left(300 \mathrm{MHz}, \mathrm{CDCl}_{3}\right)$ : 7.307.34 (m, 6 H, Ar-H), 7.35-7.41 (m, 7 H, Ar-H), 7.54-7.60 (m, 3 H, Ar-H), 7.87 (d, $J=$<smiles>c1ccc(-c2cc(-c3ccccc3)nc(-c3ccccc3)n2)cc1</smiles>
1.4, 1 H, CH), 7.95-7.99 (m, 4 H, Ar-H). ${ }^{13}$ C-NMR (75 MHz, CDCl $)$ : $109.6(\mathrm{CH}), 127.2(\mathrm{CH}), 128.1$ $\left(\mathrm{d}, J_{\mathrm{PC}}=7.6, \mathrm{CH}\right), 128.8(\mathrm{CH}), 128.8(\mathrm{CH}), 130.8(\mathrm{CH}), 134.7\left(\mathrm{~d}, J_{\mathrm{PC}}=19.8, \mathrm{CH}\right), 136.1\left(\mathrm{~d}, J_{\mathrm{PC}}=8\right.$, C), 137.0 (C), $160.8(\mathrm{C}), 163.5$ (d, $\left.J_{\mathrm{PC}}=7, \mathrm{C}\right) .{ }^{31} \mathbf{P}-\mathbf{N M R}\left(121 \mathrm{MHz}, \mathrm{CDCl}_{3}\right): 1.4$ (s). IR (KBr): 3473 (m), 3057 (m), 1565 (s), 1510 (s), 1432 (s), 749 (s), 688 (s). MS (EI, 70 eV): 416.1 (100)[M ], 339.1 (31), 233.1 (14), 183.0 (13). EA calcd for $\mathrm{C}_{28} \mathrm{H}_{21} \mathrm{~N}_{2} \mathrm{P}: \mathrm{C}$ 80.75, H 5.08, N 6.73, found C 80.67, H 5.28, N 6.72. 


\section{New Substances from Alkyne Hydration}

Known substances: 4-tert-Butylphenyl-acetaldehyde, ${ }^{5}$ tert-butyl 2-(3-oxo-propyl)-3-oxo-butanoate, ${ }^{6}$ dimethyl 2-(3-oxopropyl)-malonate, ${ }^{7}$ furthermore octanal, heptanal, phenylacetaldehyde (without reference).

4,4-dimethyl-6-oxo-heptanal (Table 3, entry 10). Prepared according to the general procedure. Colorless liquid of pleasant smell. ${ }^{\mathbf{1}} \mathbf{H}-\mathbf{N M R}$ (400 MHz,

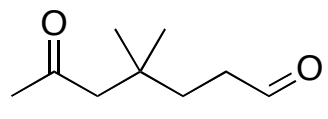
$\left.\mathrm{CDCl}_{3}\right): 1.01(\mathrm{~s}, 6 \mathrm{H}), 1.65-1.73(\mathrm{~m}, 2 \mathrm{H}), 2.14(\mathrm{~s}, 3 \mathrm{H}), 2.35(\mathrm{~s}, 2 \mathrm{H}), 2.38-2.44(\mathrm{~m}, 2 \mathrm{H}), 9.77(\mathrm{t}, \mathrm{J}=$ 1.8, H). ${ }^{13}$ C-NMR (100 MHz): $27.1\left(\mathrm{CH}_{3}\right), 32.5\left(\mathrm{CH}_{3}\right), 33.0(\mathrm{C}), 33.4\left(\mathrm{CH}_{2}\right), 39.4\left(\mathrm{CH}_{2}\right), 53.5\left(\mathrm{CH}_{2}\right)$, 202.4 (C), 208.3 (C). IR (film): 2984w, 1684s, 1651m, 1559m, 1457m, 1266m, 754m, 668m. MS (EI): 156 (1, M+), 138 (12), 123 (12), 113 (55), 81 (48), 69 (40), 43 (100). EA: The analytical sample was oxidized to the carboxylic acid on standing in air; calcd for $\mathrm{C}_{9} \mathrm{H}_{16} \mathrm{O}_{3}: \mathrm{C} 62.77, \mathrm{H} 9.36$, found $\mathrm{C} 62.88$, H 9.51 .

4-phenyl-4-pivaloyloxy-butanal (Table 3, entry 11). Colorless oil. ${ }^{\mathbf{1}} \mathbf{H}-\mathbf{N M R}$ (300 $\left.\mathrm{MHz}, \mathrm{CDCl}_{3}\right): 1.21(\mathrm{~s}, 9 \mathrm{H}), 2.07-2.28(\mathrm{~m}, 2 \mathrm{H}), 2.47(\mathrm{t}, \mathrm{J}=7.5,2 \mathrm{H}), 5.74(\mathrm{dd}, \mathrm{J}=\mathrm{Ph}$ 7.2, 6.1, $1 \mathrm{H}), 7.24-7.40(\mathrm{~m}, 5 \mathrm{H}), 9.73$ (t, J = 1.3, $1 \mathrm{H}) .{ }^{13} \mathbf{C}-\mathbf{N M R}\left(75 \mathrm{MHz}, \mathrm{CDCl}_{3}\right): 27.1\left(\mathrm{CH}_{3}\right), 29.0$ $\left(\mathrm{CH}_{2}\right), 38.8(\mathrm{C}), 39.9\left(\mathrm{CH}_{2}\right), 74.6(\mathrm{CH}), 126.0(\mathrm{CH}), 128.0(\mathrm{CH}), 128.6(\mathrm{CH}), 140.2(\mathrm{C}), 177.5(\mathrm{C})$, 201.0 (C). IR (film): 3022w, 2976w, 1718m, 1216m, 1157m, 760s. MS (EI+): 248 (1, M+), 204 (40), 163 (100), 147 (67), 129 (50), 117 (85), 91 (90). EA calcd for $\mathrm{C}_{15} \mathrm{H}_{20} \mathrm{O}$ (248.32): C 72.55, H 8.12, found $\mathrm{C} 72.00, \mathrm{H}$ 8.04; calcd for $\mathrm{M}+0.1 \mathrm{O}$ (partial oxidation to carboxylic acid): C 72.09, H 8.07. 


\section{Selected NMR Spectra}

6.1. Ligand Synthesis

${ }^{1}$ H NMR of P6

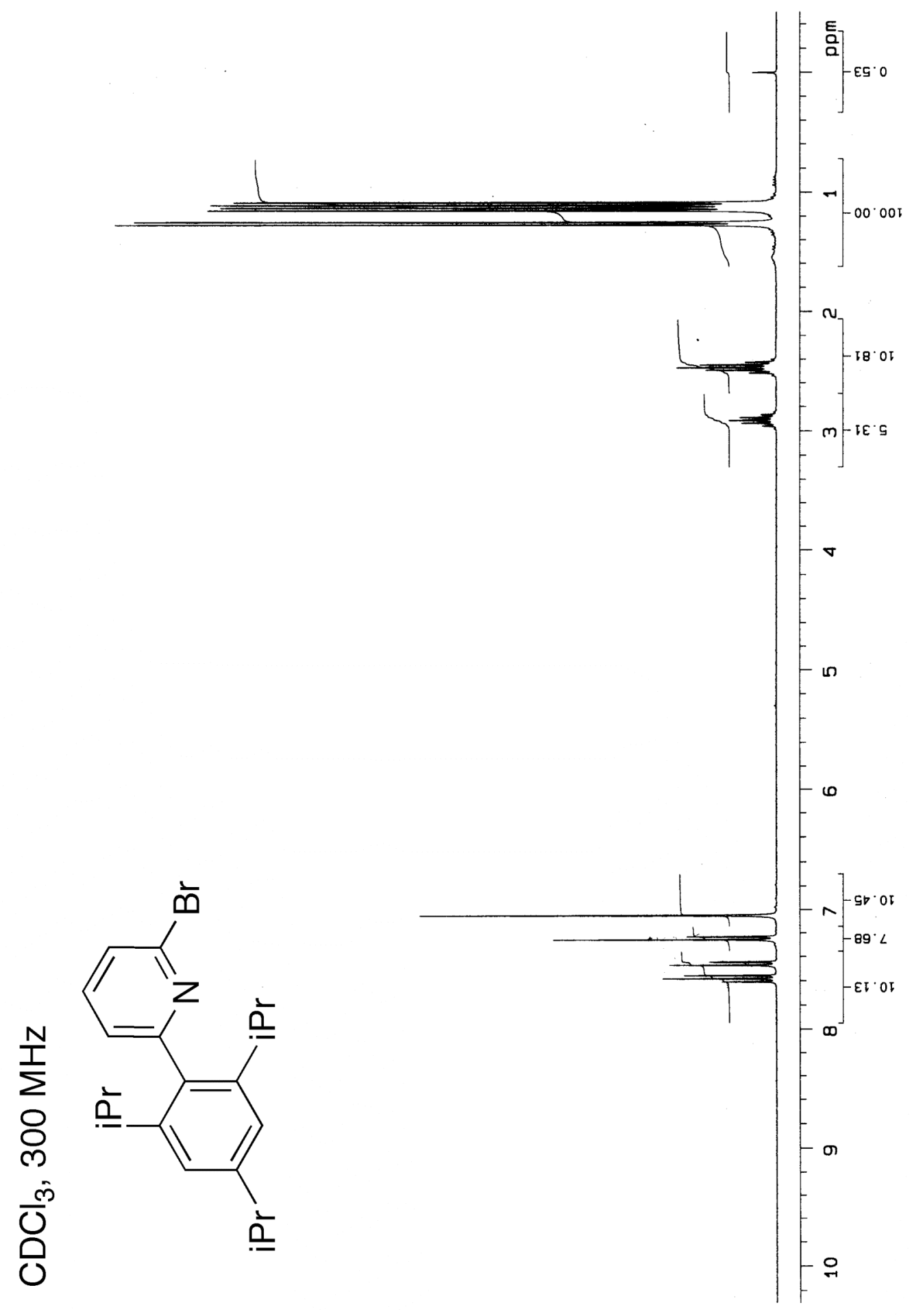


${ }^{13} \mathrm{C}$ NMR of $\mathbf{P 6}$

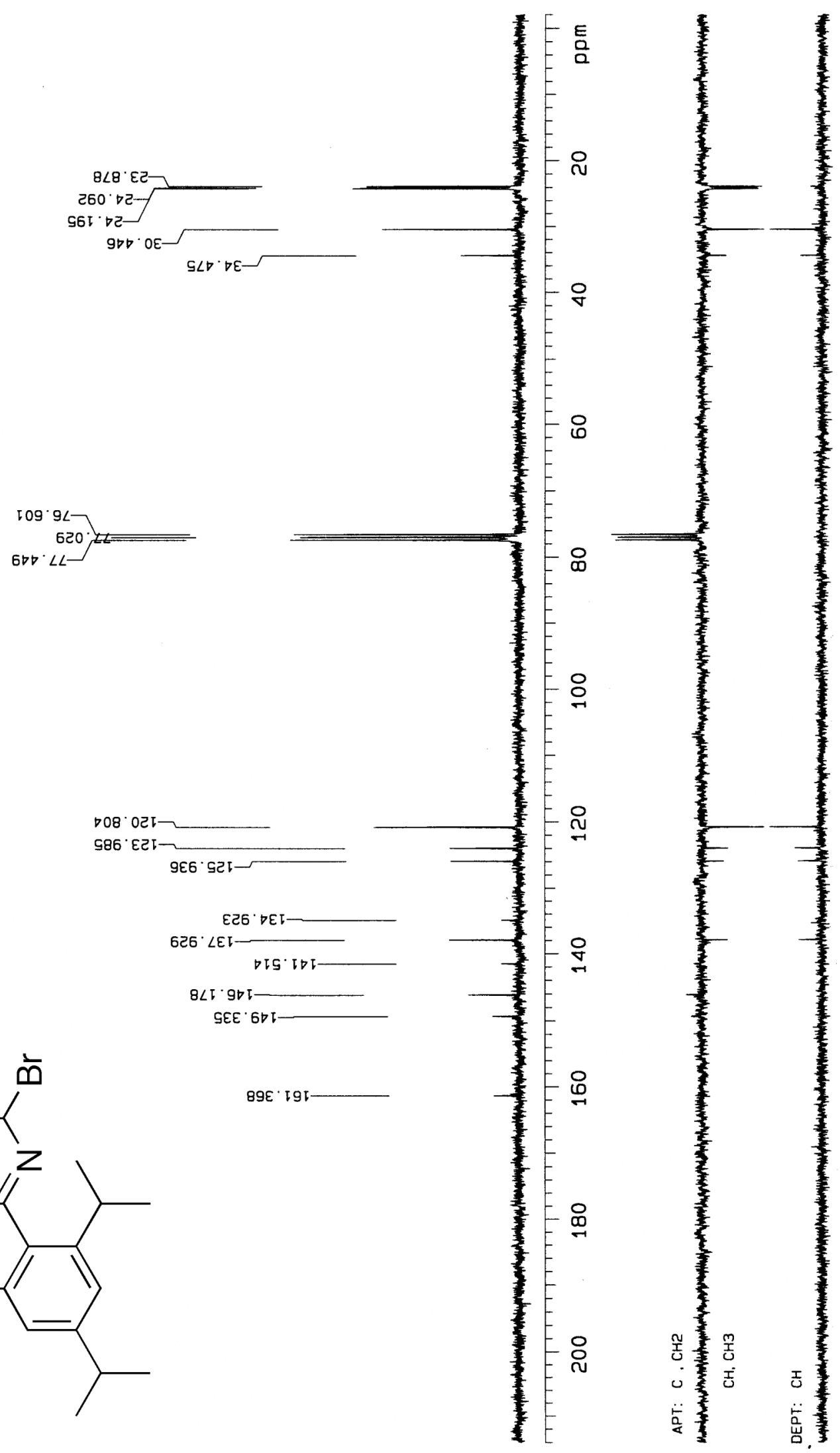


${ }^{1} \mathrm{H}$ NMR of P7

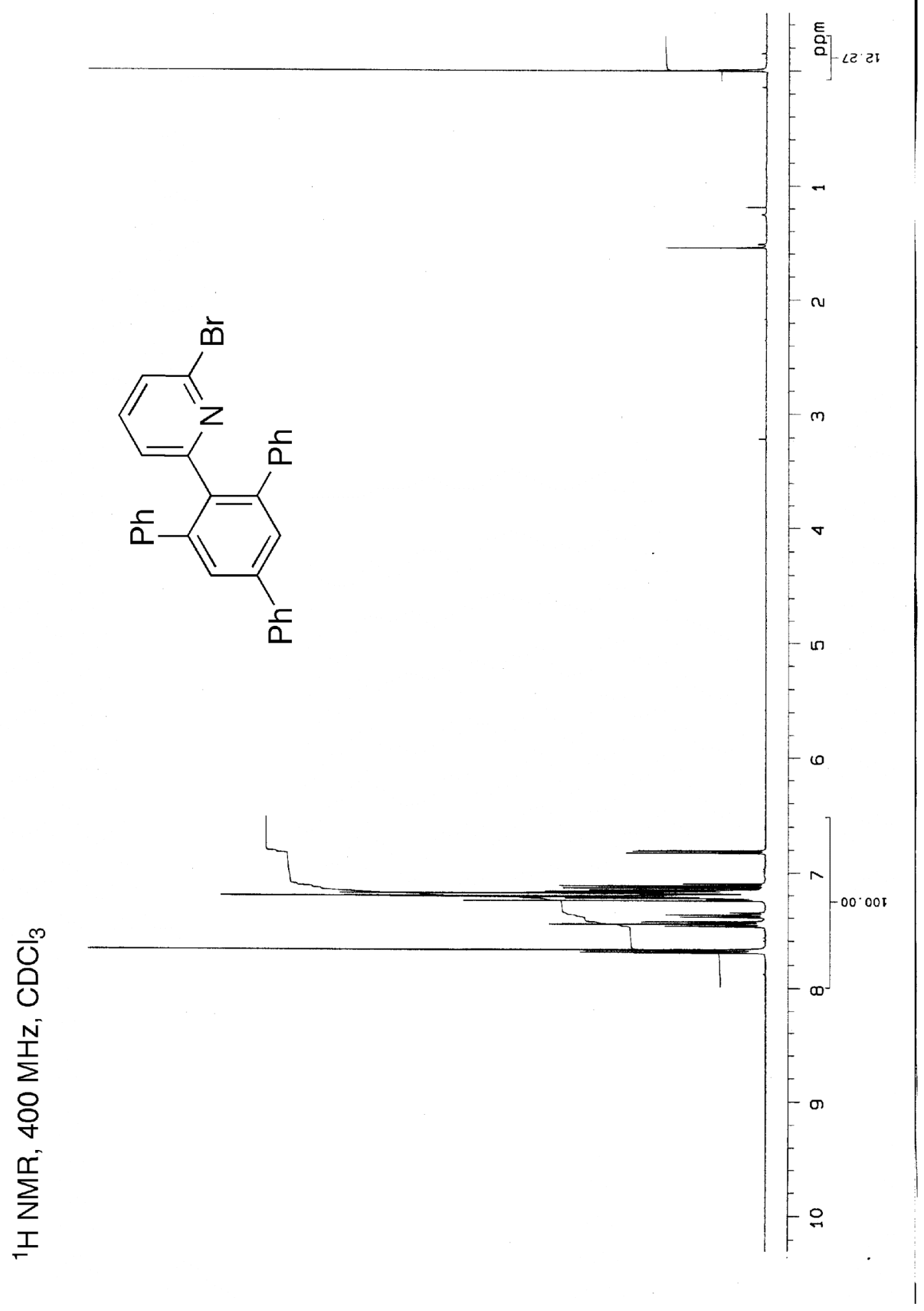


${ }^{1} \mathrm{H}$ NMR of L5

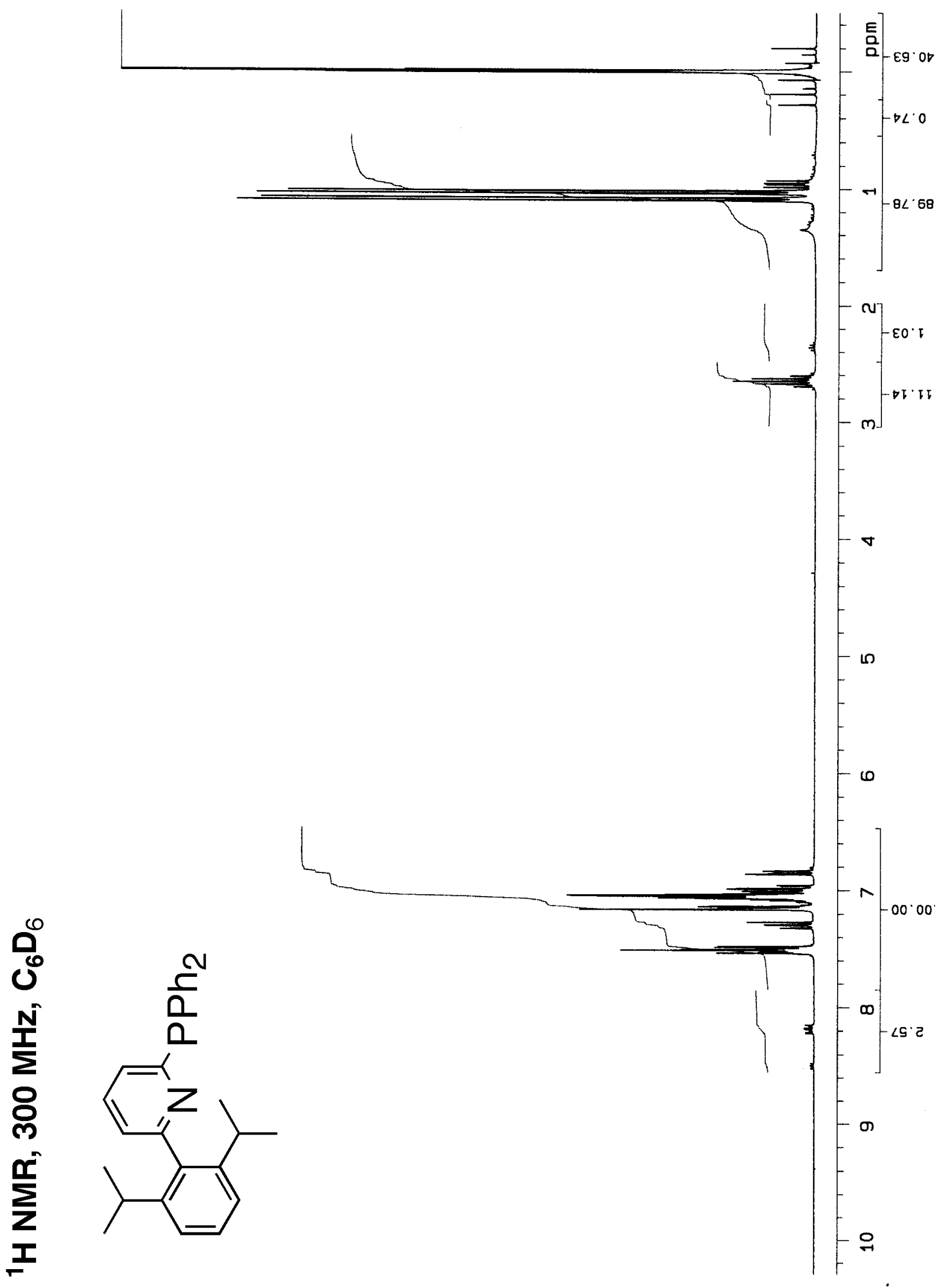

(traces of phosphine oxide due to NMR sample oxidation) 
${ }^{1} \mathrm{H}$ NMR of $\mathbf{L 6}$

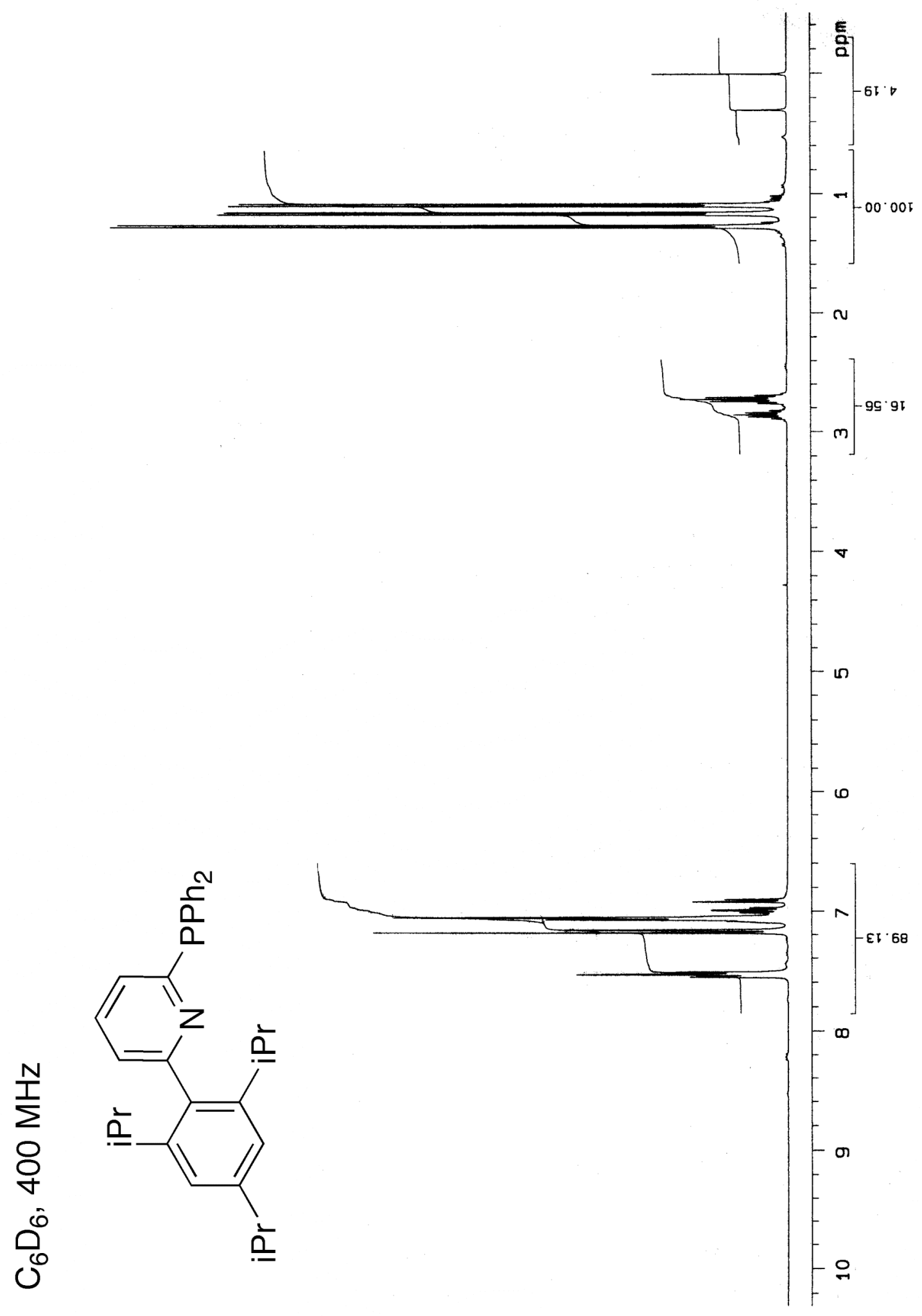


${ }^{1} \mathrm{H}$ NMR of $\mathbf{L} 7$

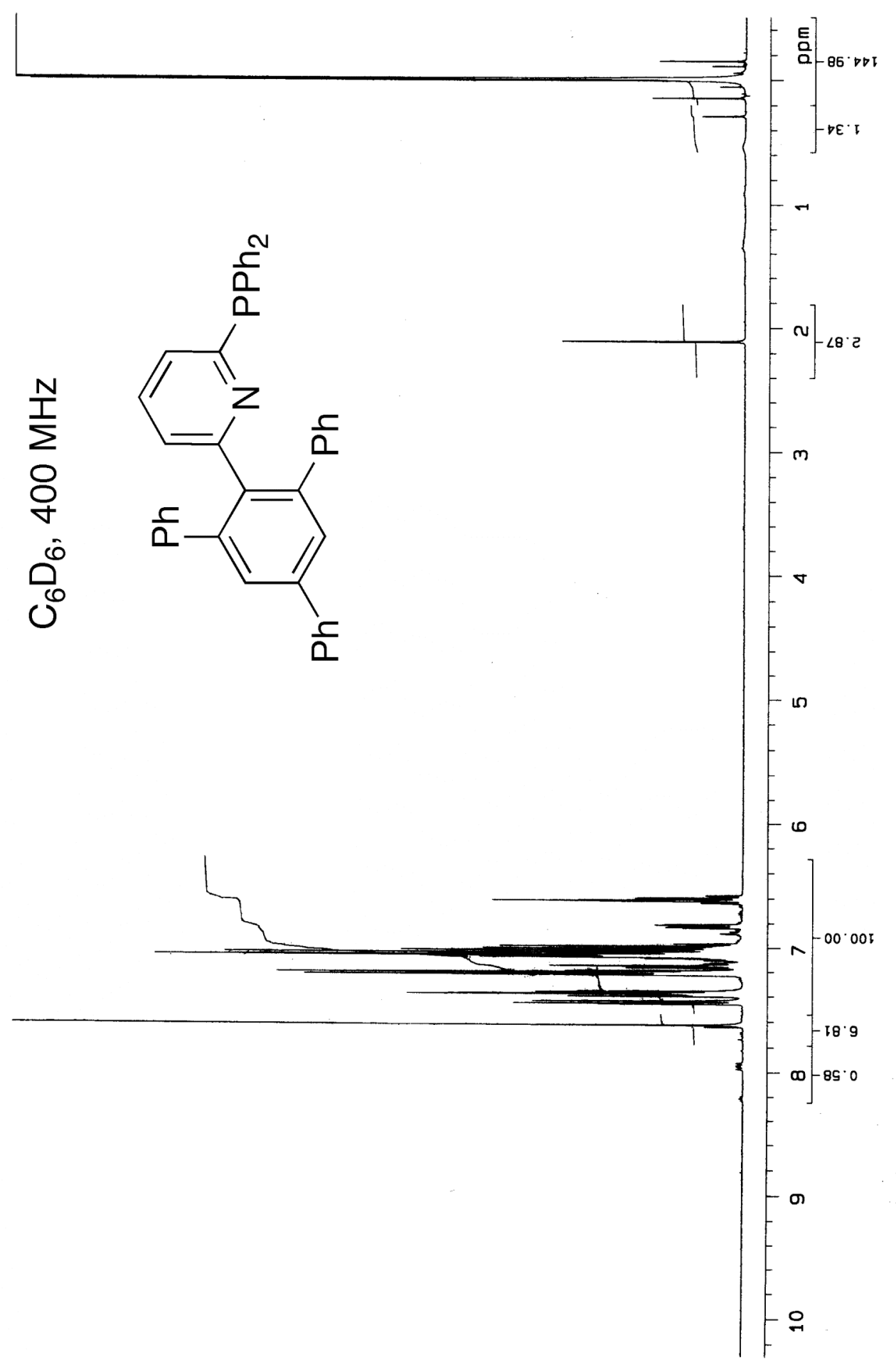


${ }^{31}$ P NMR of L6, L7

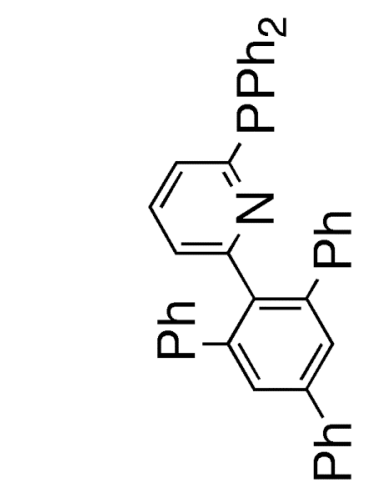

โEE'

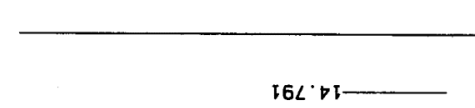

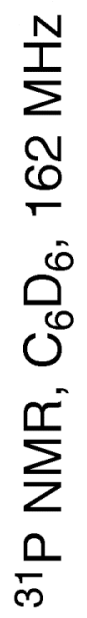

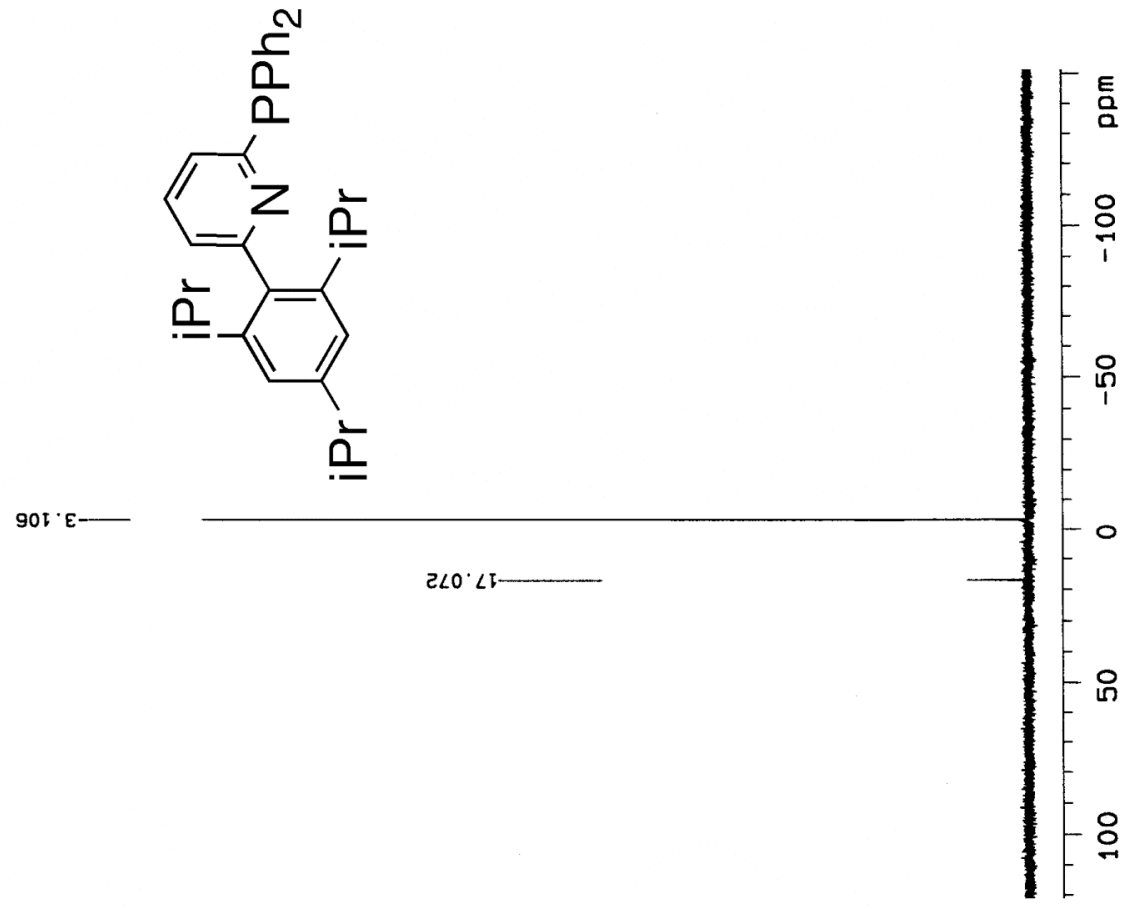

Traces of phosphine-oxide due to NMR sample oxidation in air. 
6.2. Hydration Products (Table 1 of the main article)

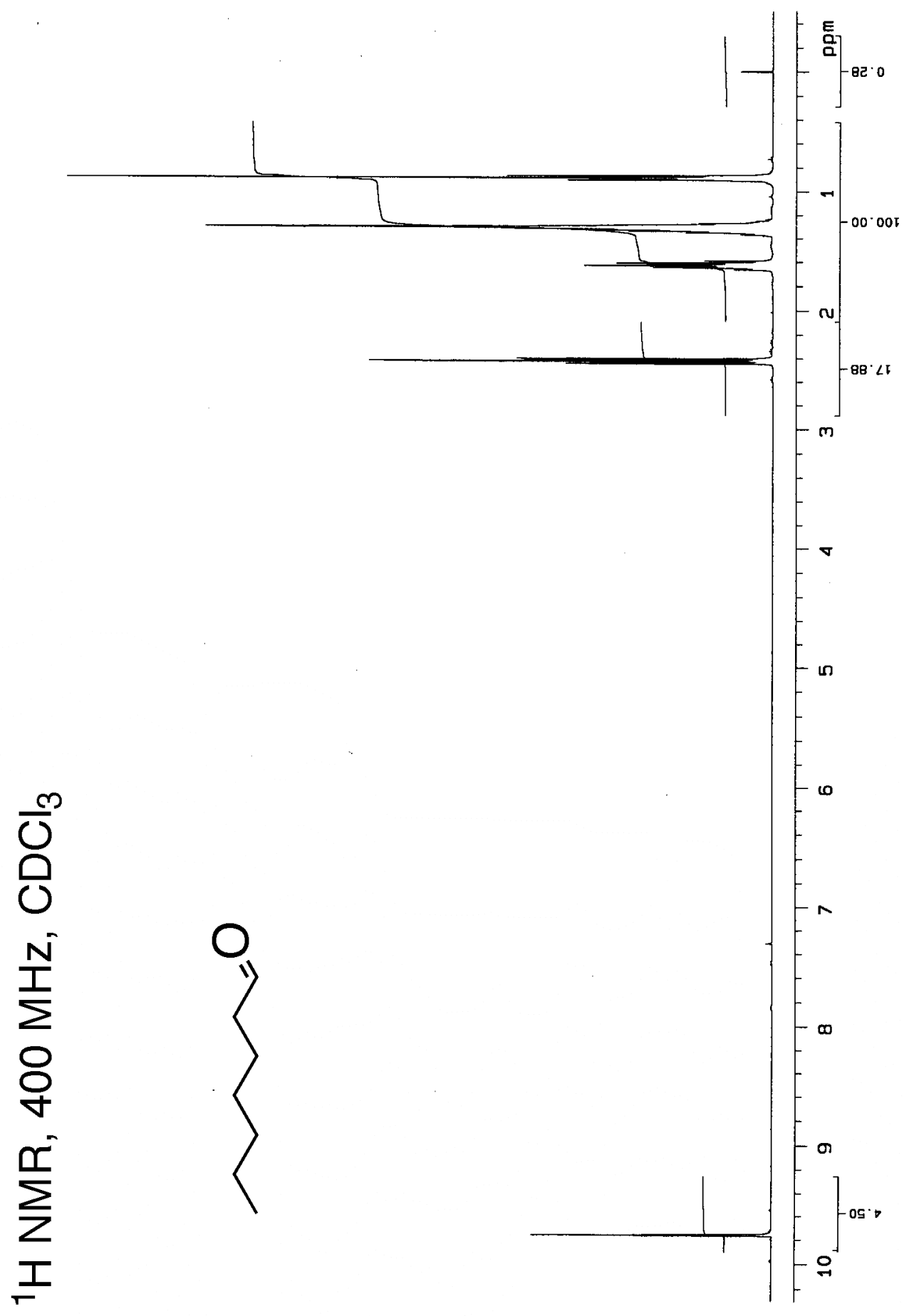




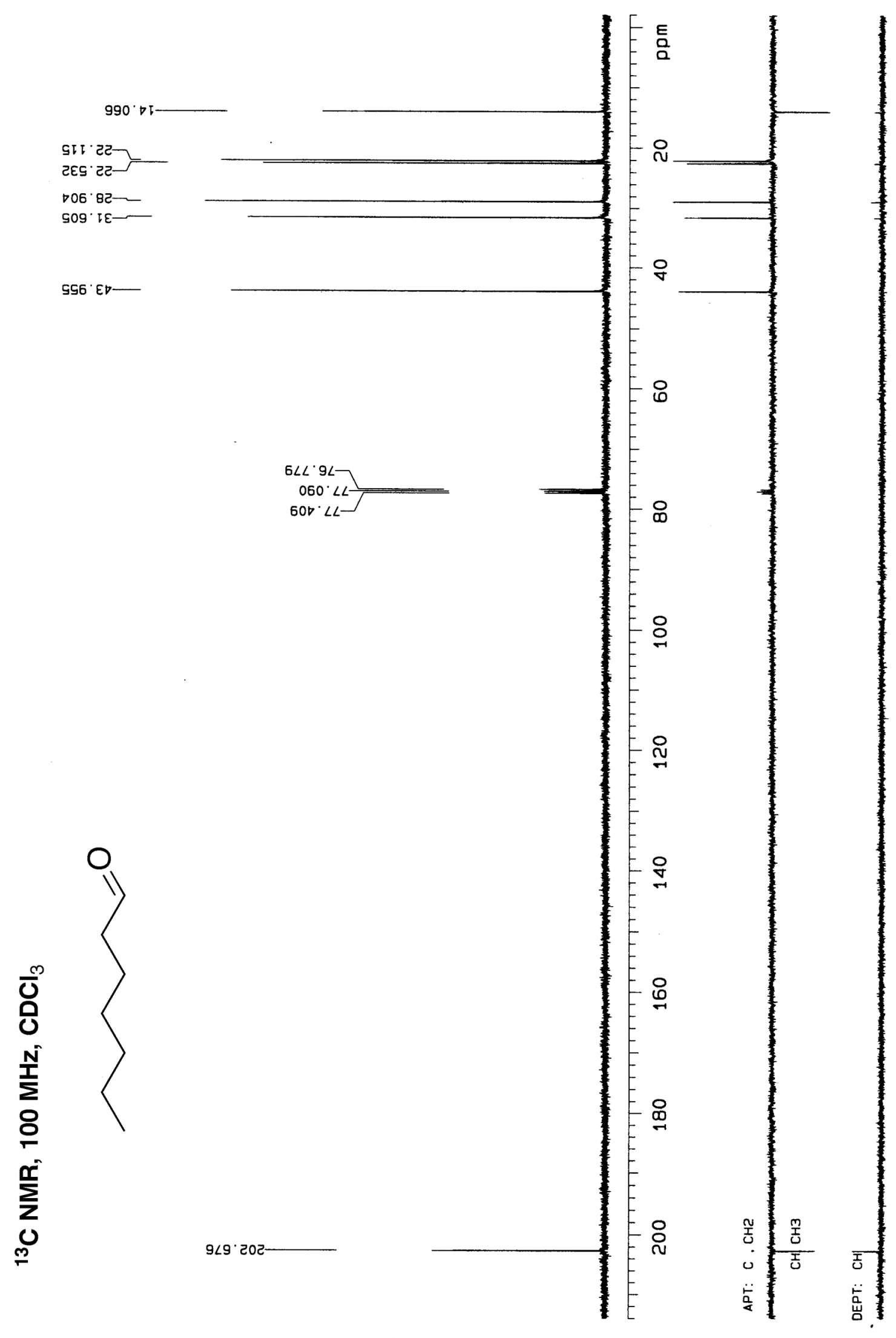




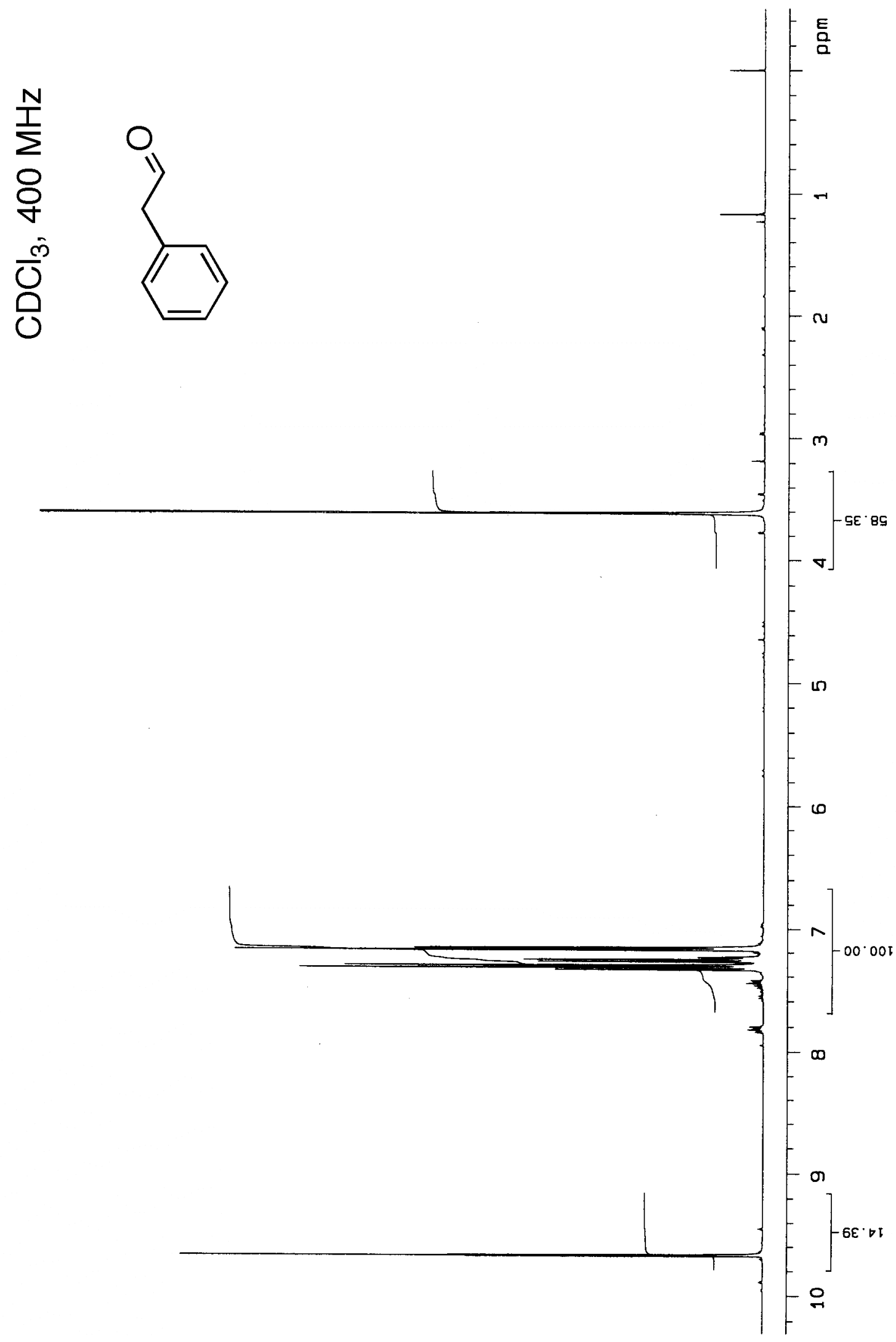




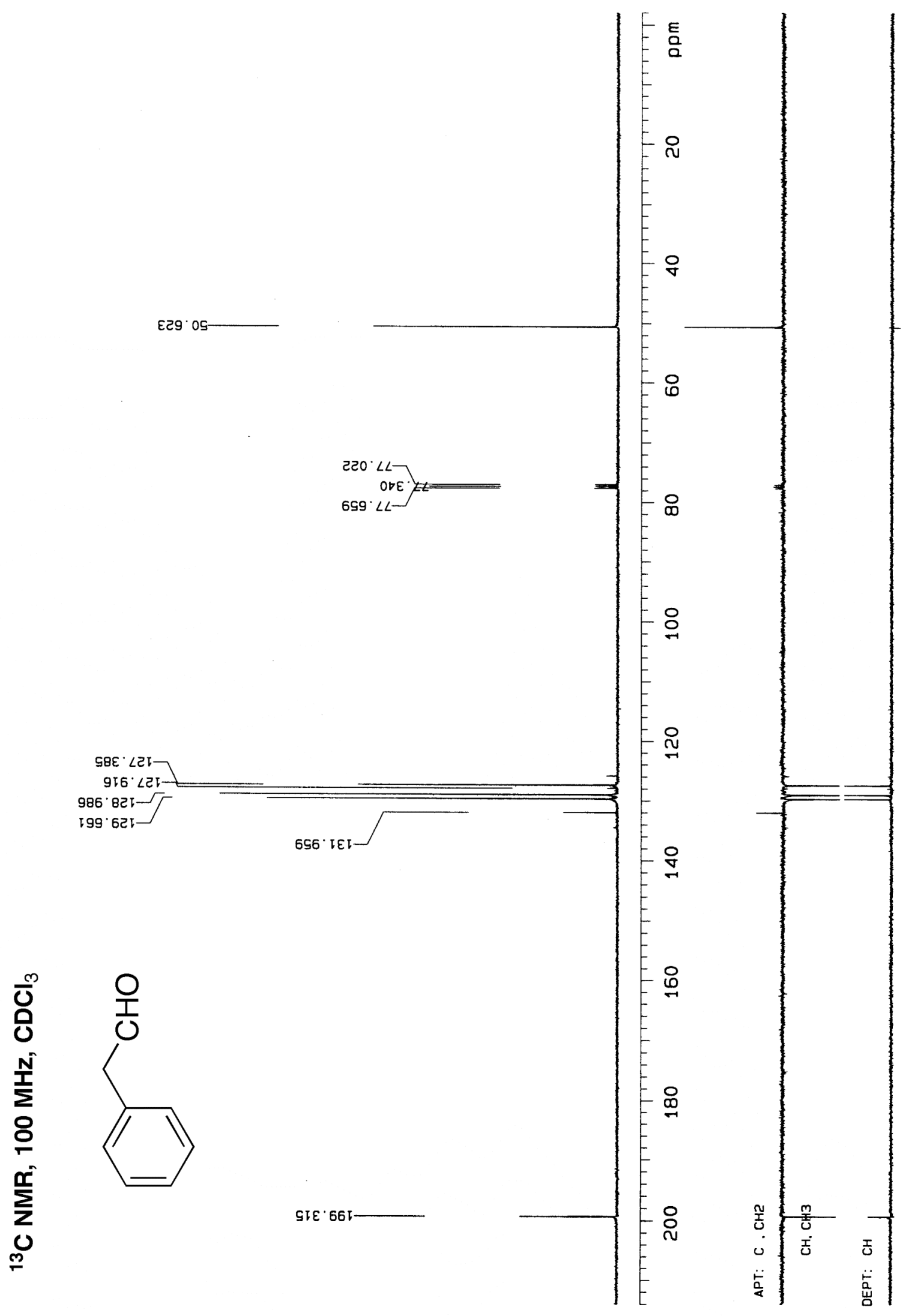




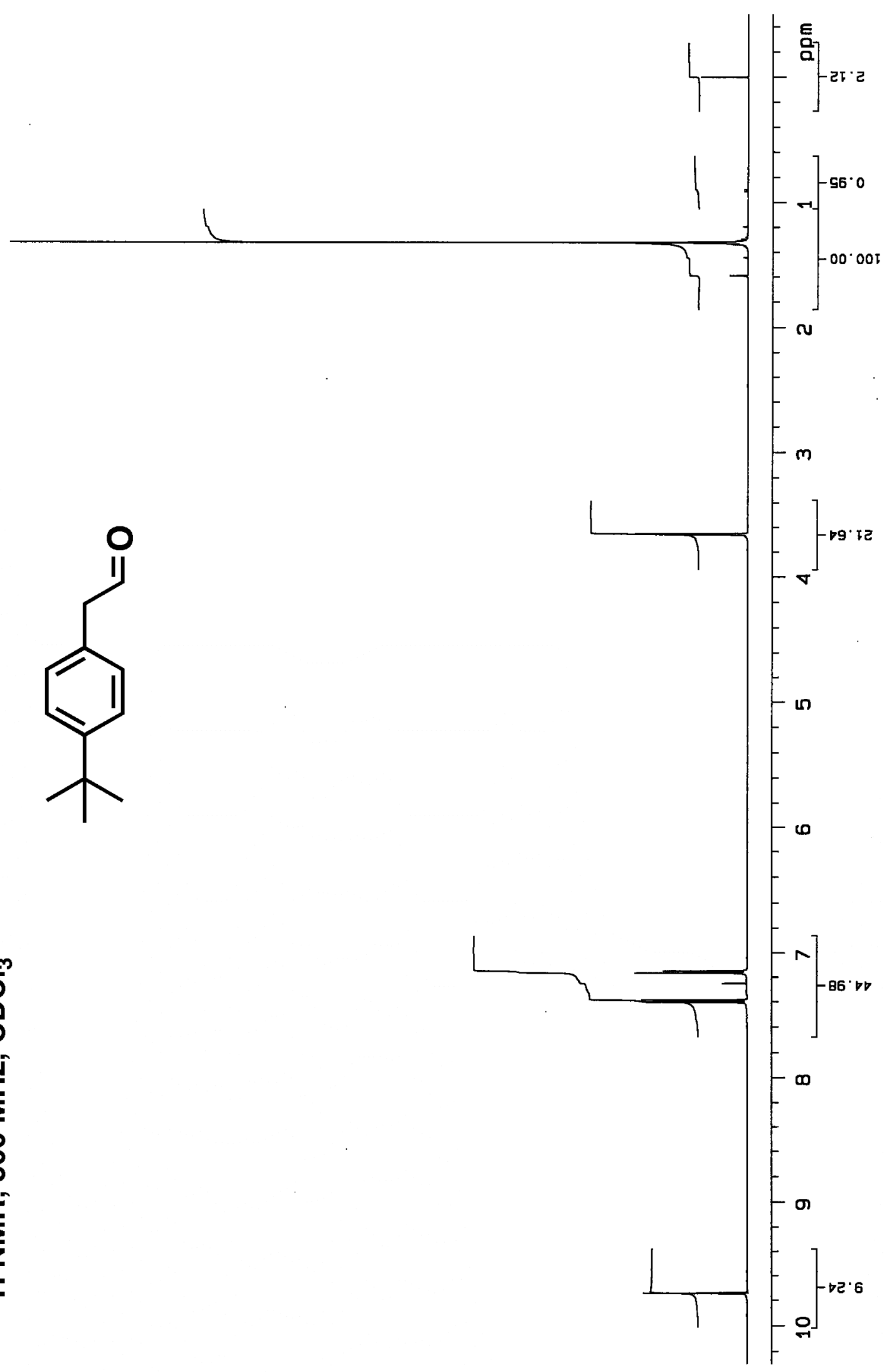




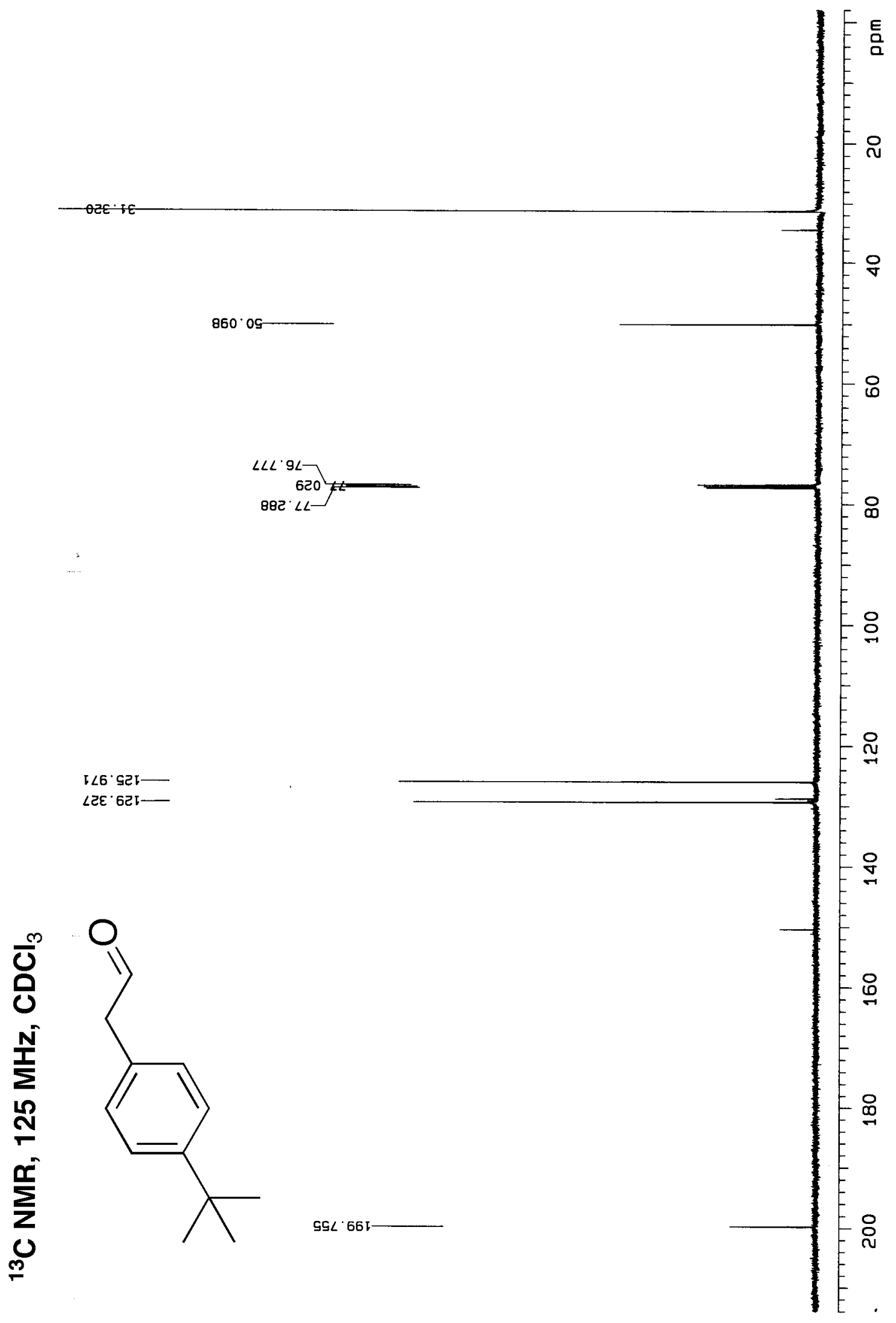




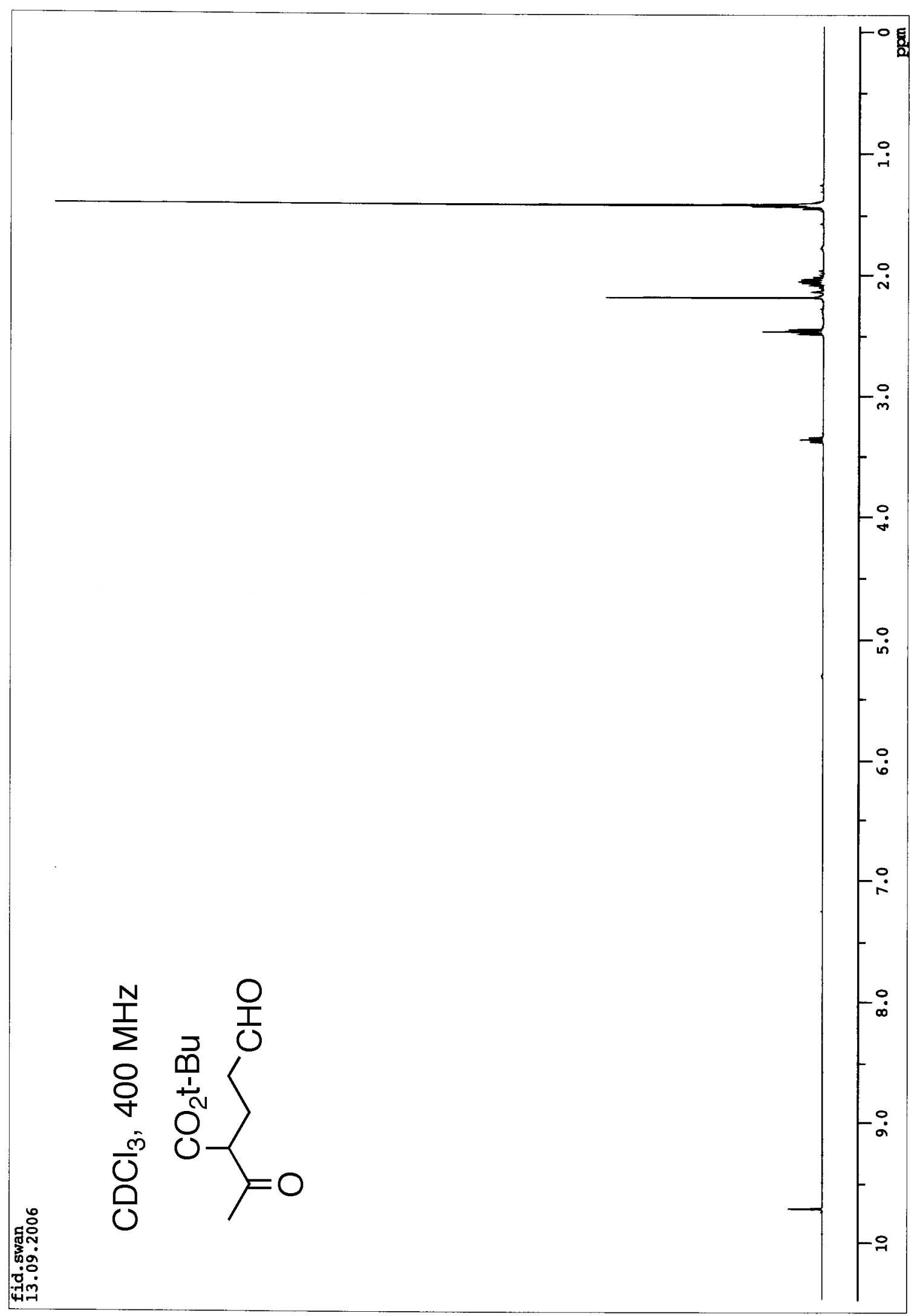




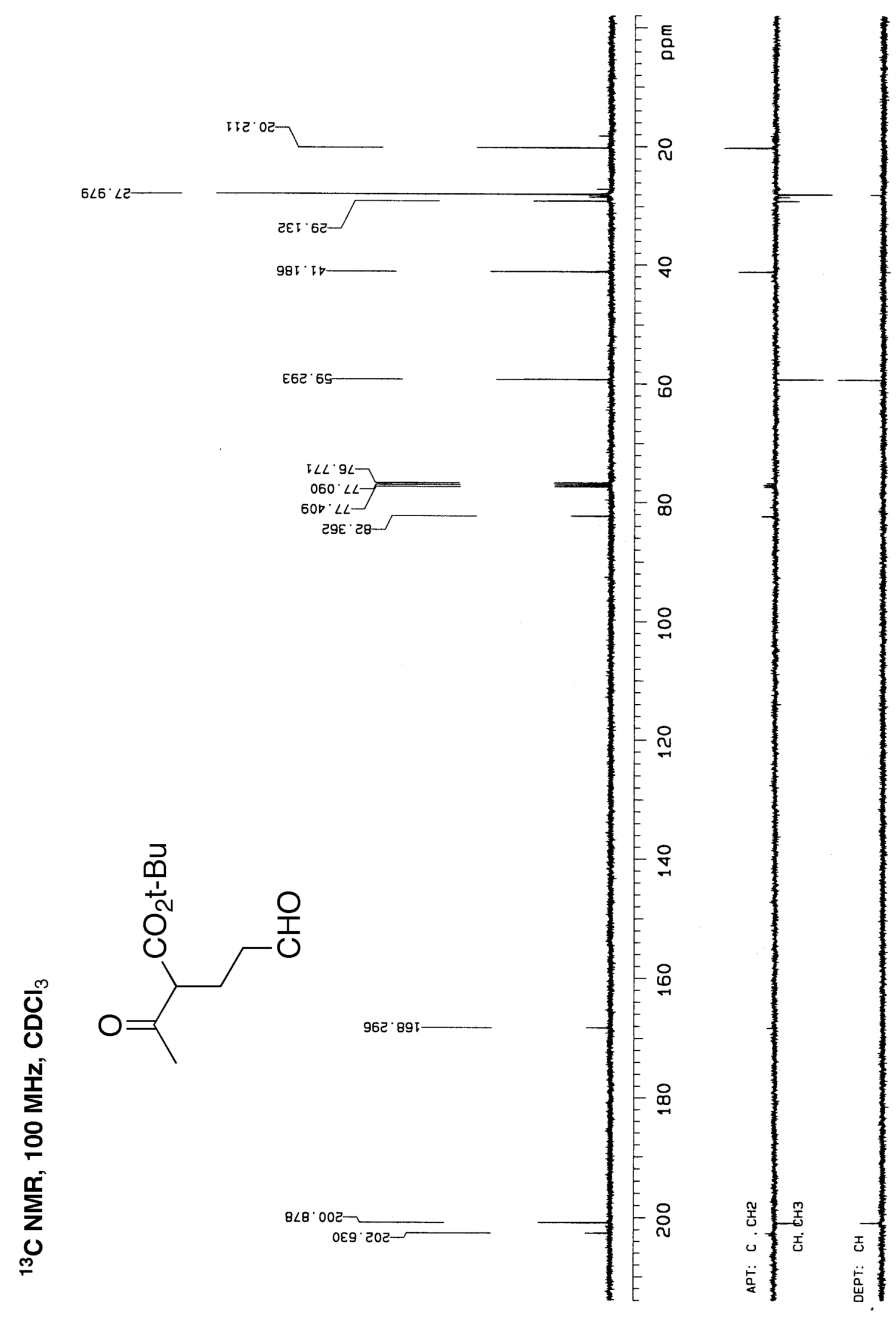




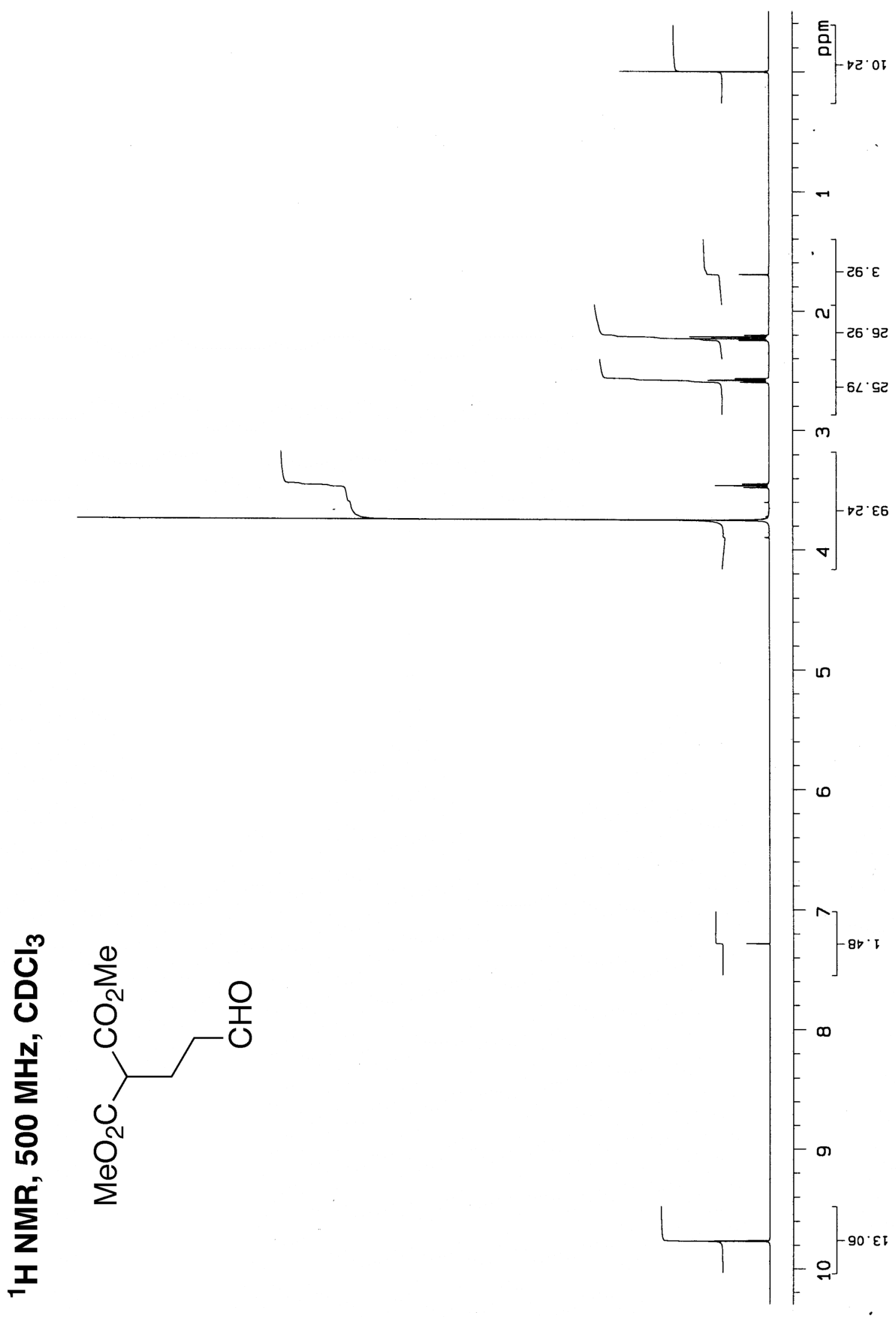




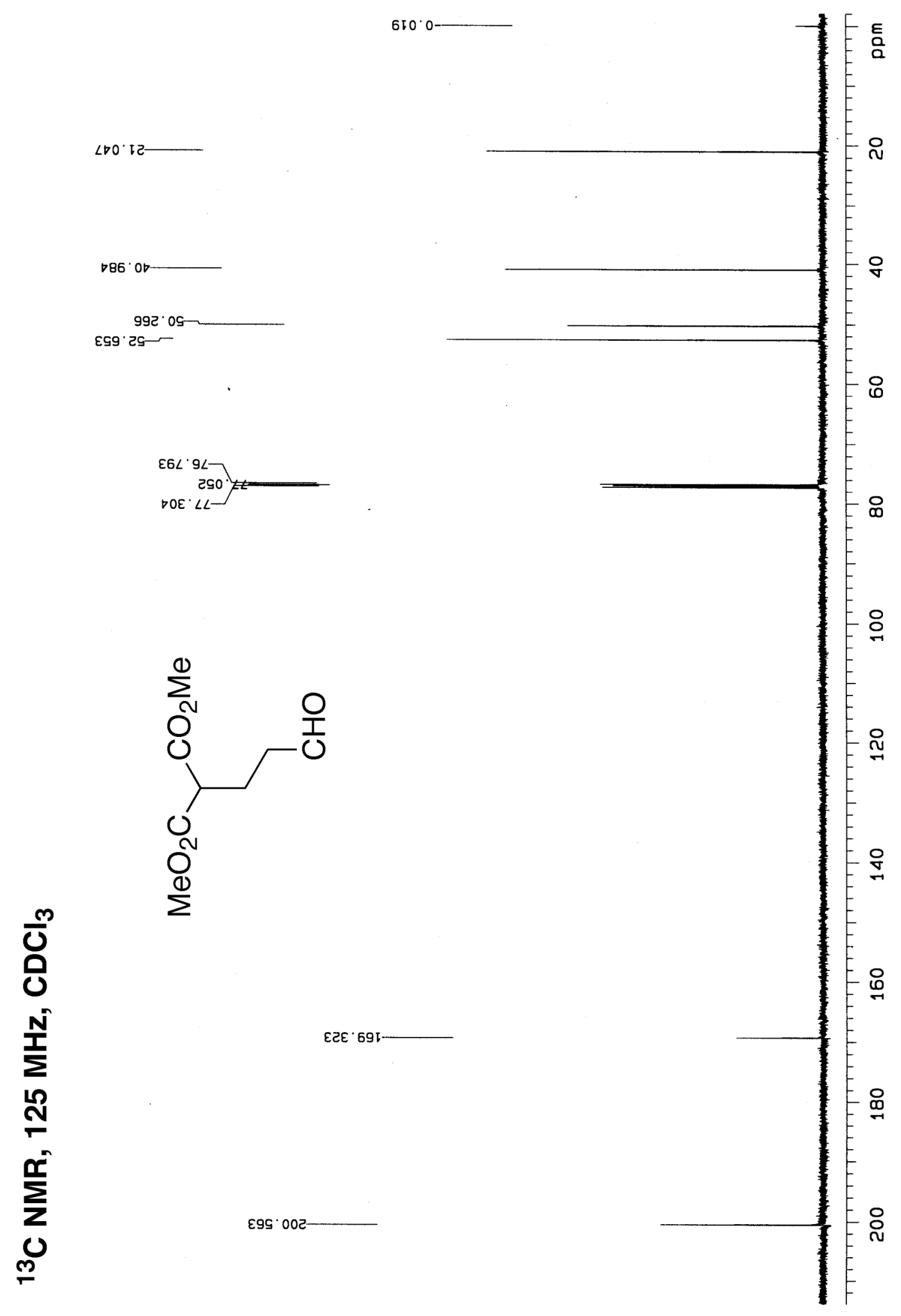




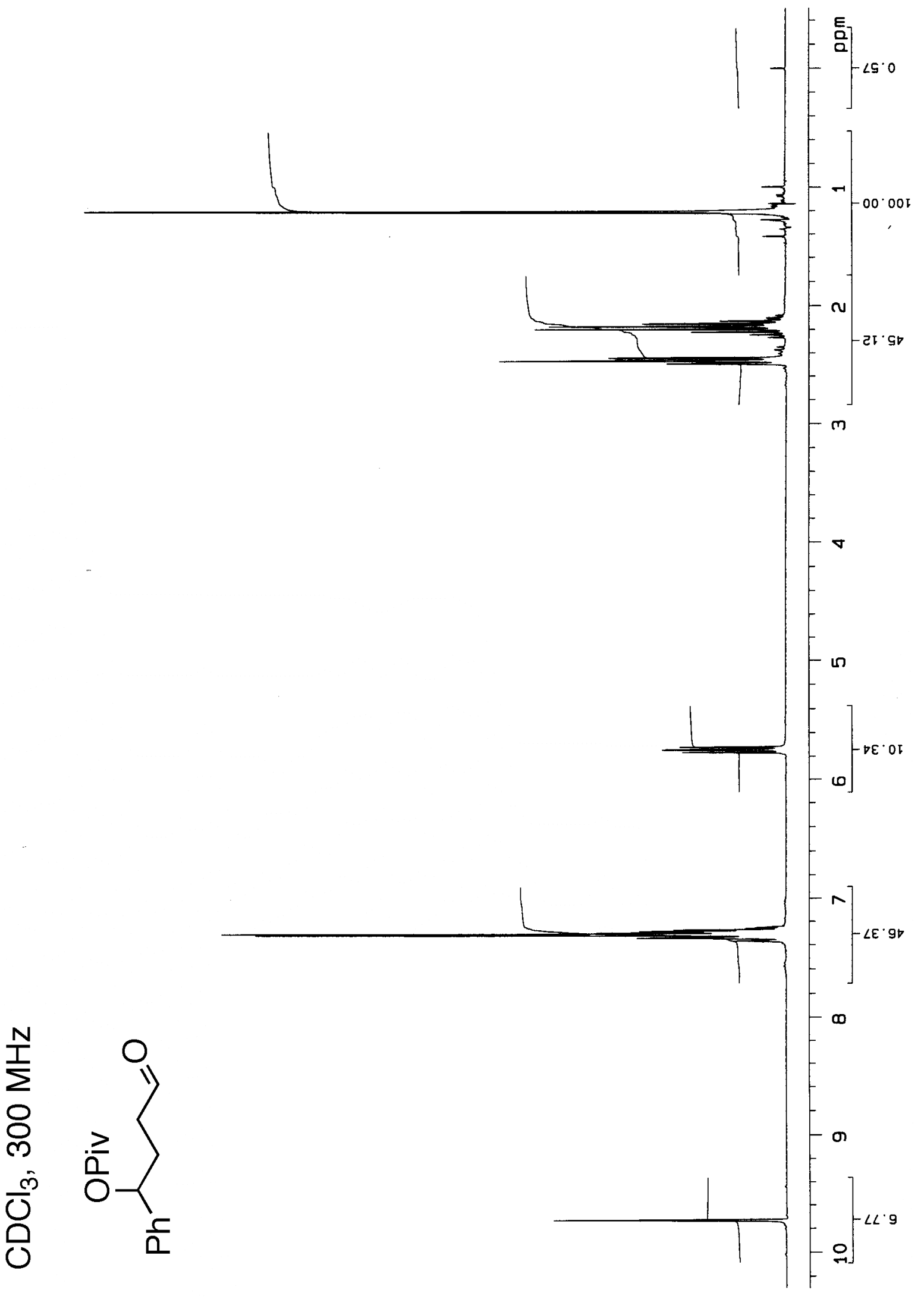




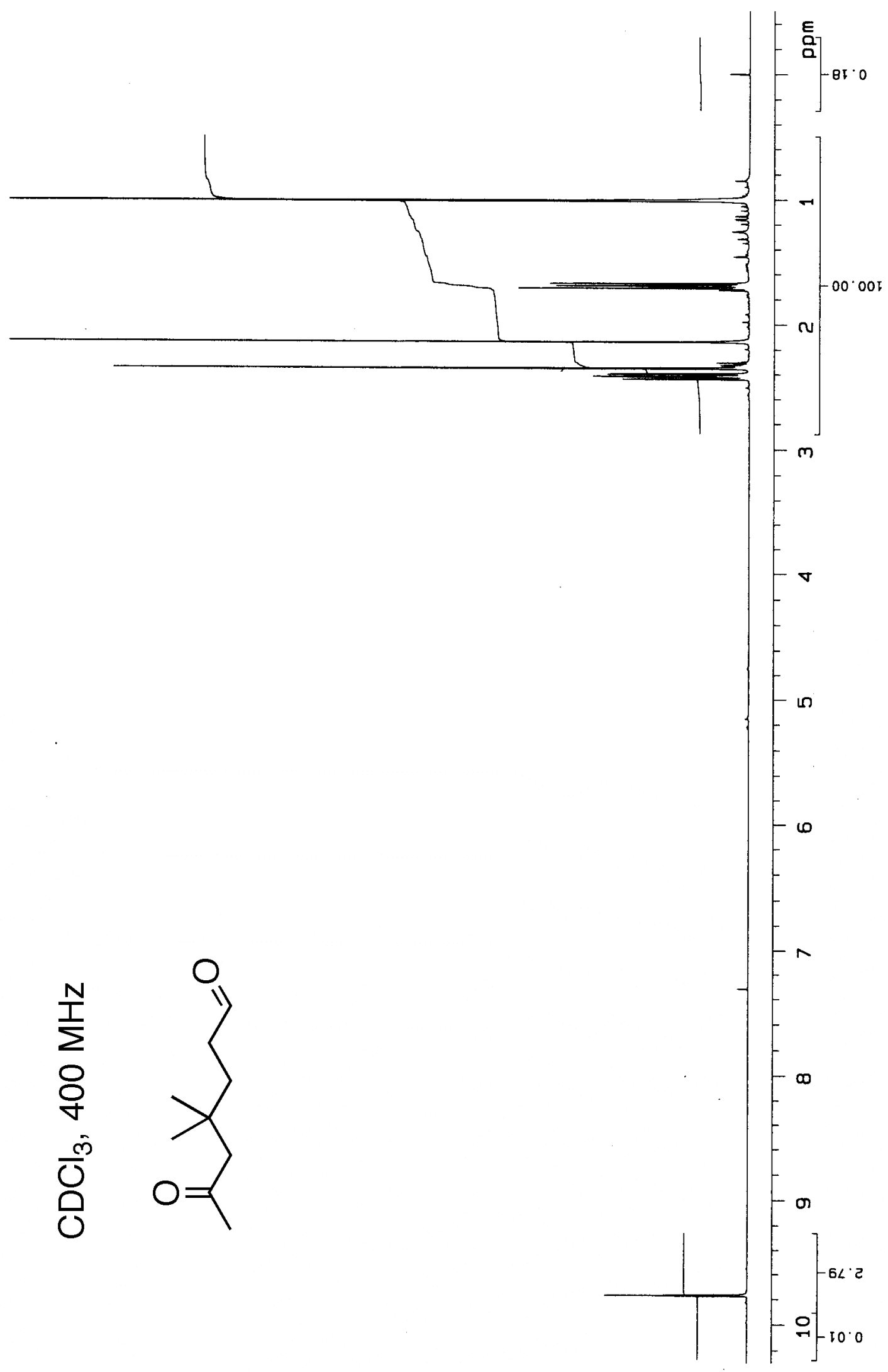




\section{References}

${ }^{1}$ Nasielski, J.; Standaert, A.; Nasielski-Hinkens, R. Synth. Commun. 1991, 21, 901.

${ }^{2}$ Gros, P.; Fort, Y. J. Org. Chem. 2003, 68, 2028.

${ }^{3}$ Hewertson, W.; Watson, H. R. J. Chem. Soc. 1962, 1490.

${ }^{4}$ Scott, N. M.; Schareina, T.; Tok, O.; Kempe, R. Eur. J. Inorg. Chem. 2004, 3297.

${ }^{5}$ Geier, G. R.; Sasaki, T. Tetrahedron 1999, 55, 1859.

${ }^{6}$ Bartoli, G.; Bosco, M.; Bellucci, M. C.; Marcantoni, E.; Sambri, L.; Torregiani, E. Eur. J. Org. Chem. 1999, 617.

${ }^{7}$ Fournet, G.; Balme, G.; Barieux, J. J.; Gore, J. Tetrahedron 1988, 44, 5821. 